\title{
The characters of some induced representations of semisimple Lie groups
}

\author{
By \\ Takeshi HiraI \\ (Received February 9, 1968)
}

\section{Introduction.}

Let $G$ be a connected semisimple Lie group. A general method to construct representations of $G$ is to induce, in a sense, representations of some subgroups of $G$. In particular, all finite-dimensional irreducible representations of $G$ are obtained by inducing one-dimensional representations of a certain subgroup. But the situation is not so simple in the case of infinite-dimensional representations. Concerning this problem, F. Bruhat obtained a criterion for irreducibility of some induced representations of $G$ in [1]. The purpose of the present paper is to give a formula which expresses the characters of such induced representations of $G$ on Hilbert spaces that has been treated by $\mathrm{F}$. Bruhat, by means of the characters of the original representations. Our principal result is Theorem 2 in $\S 5$, which gives this formula. This formula generalizes analogous ones for very particular cases considered in [2], [3], and [4(c)]. In the opinion of the author, this formula has an important significance for such problems to establish the Plancherel formula for $G$, to obtain the characters of all (quasi-simple) irreducible representations of $G$ on Hilbert spaces, and to exhaust all invariant eigendistributions of all Laplace operators on $G$ (see for example [5(a), (b), (c)]).

As an application of our result, the characters of all unitary irreducible representations of $G L(3, \boldsymbol{R})$ and $S L(3, \boldsymbol{R})$ can be calculated 
explicitely by using the result [10] of I. Ya. Vakhutinskii (see the last note in $\$ 5$ ).

Also, in the forth coming paper $[4(\mathrm{~d})]$, we obtain the explicite form of the Plancherel formula for $S U(p, q)$ by using the result of this paper (Theorem 2) and the result of $[4(\mathrm{c}), \S 10]$ on the explicite form of the characters of irreducible unitary square-integrable representations of $S U(p, q)$. $^{1)}$

Section 1 is devoted to construct induced representatiors $T^{L}$ of some representations $L$ of a certain subgroup $S_{1}$ of $G$ in such a form that are convenient for our purpcse. In this section, some elementary facts are stated without proofs for the sake of brevity, but the reader can fird their prcofs in another papers cited in places. In Section 2, we study a sufficient cordition for the existerce of the characters of the representaticns $L$ ard $T^{L}$. In Section 3 , we prove an integral formula on $G$ which is essential to calculate explicitely the character of $T^{L}$ in Section 5. Let $x(g)$ ke an indefiritely differentiable function on $G$ which vanishes outside a compact set and put

$$
T_{a:}^{L}=\int_{G} T_{g}^{L} x(g) d g
$$

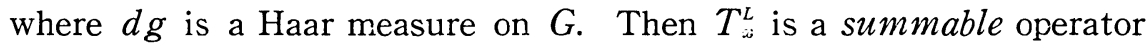
under certain conditions on $S_{1}$ and $L$ (for the definition, see $\$ 2$ ). The principal result in Section 4 is to express the trace $\operatorname{Sp}\left(T_{x}^{L}\right)$ of $T_{x}^{L}$ by means of an integral on $G$ containing $x$ and the character $\tau$ of $L$ (Theorem 1). In other words, the character $\pi$ of $T^{L}$ is expressed as a distribution on $G$ defined from $\tau$. To prove Theorem 1, we must verify the following fact. Let $T$ be a summable integral operator on a Hilbert space of all square-integrable functions on a certain locally euclidian measure space $\left\{\mathfrak{u}, d_{\mu}\right\}$. Then the trace $\operatorname{Sp}(T)$ of $T$ is expressed as

$$
\operatorname{Sp}(T)=\int_{\mathfrak{u}} K(u, u) d \mu(u)
$$

1) $S U(p, q)$ is a real form of $S L(p+q ; \boldsymbol{C})$ consisting of all $g \in S L(p+q ; \boldsymbol{C})$ which fulfill that $g^{*} I g=1$, where $I$ is a diagonal matrix whose $p$ diagonal elements are 1 and the other $q$ elements are -1 , and $g^{*}=t \bar{g}$. 
where $K$ is the integral kernel of $T$ and $d_{\mu}$ is the measure on $\mathfrak{u}$. The last half of Section 4 is devoted to prove this fact in more general form than necessary at the time. In Section 5 , assuming some additional conditions on $S_{1}$ and $L$, we rewrite the expression of $\operatorname{Sp}\left(T_{x}^{L}\right)$ in Theorem 1 in more simpler form and thus we obtain our principal result, a simple formula expressing the character $\pi$ of $T^{L}$ by means of the character $\tau$ of $L$ (Theorem 2). In the last note of the section, the essential pcints of the proof of our formula are summalized.

The formulas (4.12) in Theorem 1 and (5.9) in Theorem 2 which express $\pi$ by means of $\tau$ may hold for invariant eigendistributiors on the grours $S_{1}$ and $G$. That is, if we take any invariant eigerdistribution $\tau$ on $S_{1}$ of all Laplace operators on it and define a distribution $\pi$ on $G$ by the formula (4.12) or the one (5.9). Then, under some appropriate conditions on $S_{1}, \pi$ may be well-defined and give an invariart eigendistribution on $G$ of all Laplace operators on it. In $[5(\mathrm{c}), \S 7]$, we prove this fact and use it to obtain a complete system of linearly inderendent invariant eigendistributions on $G$ of all Laplace operators, for $G=S U(p, q)(p, q \geqslant 1)$.

The author expresses his hearty thanks to Professor H. Yoshizawa for his kind advices and to Dr. N. Tatsuuma and Mr. S. Andô for their aids to prepare the manuscript.

Short summary of the results of this paper has been published in $[5(\mathrm{e})]$.

\section{§1. Some induced representations.}

1. Let $G$ be a simply connected semisimple Lie group whose Lie algebra is denoted by $\mathfrak{g}_{0}$. Let $\mathfrak{g}_{0}=\mathfrak{p}_{0}+\mathfrak{f}_{0}$ be a Cartan decomposition of $\mathfrak{g}_{0}$, where as usual $\mathfrak{f}_{0}$ denotes a maximal compact subalgebra. Let $\mathfrak{g}, \mathfrak{p}$, and $\mathfrak{t}$ be the complexifications of $\mathfrak{g}_{0}, \mathfrak{p}_{0}$, and $\mathfrak{t}_{0}$ respectively. Then $\mathfrak{g}=\mathfrak{p}+\mathfrak{f}$. Let $\mathfrak{c}_{0}$ be the center of $\mathfrak{t}_{0}$ and put $\mathfrak{t}_{0}^{\prime}=\left[\mathfrak{t}_{0}, \mathfrak{t}_{0}\right]$. Then $\mathfrak{t}_{0}=\mathfrak{t}_{0}^{\prime}+\mathfrak{c}_{0}$ and $\mathfrak{k}=\mathfrak{t}^{\prime}+\mathfrak{c}^{\prime}$ where $\mathfrak{t}^{\prime}$ and $\mathfrak{c}^{\prime}$ are the complexifications of $\mathfrak{t}_{0}^{\prime}$ and $c_{0}^{\prime}$ respectively. Let $K, K^{\prime}$, and $D$ be the analytic subgroups 
corresponding to $\mathfrak{f}_{0}, \mathfrak{f}_{0}^{\prime}$, and $\mathfrak{c}_{0}$ respectively. Let $\mathfrak{h}_{0}^{-}$be a maxinal abelian subalgebra of $\mathfrak{p}_{0}$, and let $\mathfrak{h}_{0}=\mathfrak{h}_{0}^{-}+\mathfrak{h}_{0}^{+}$be a Cartan subalgejra of $\mathfrak{g}_{0}$ where $\mathfrak{h}_{0}^{+} \subset \mathfrak{f}_{0}$. Let $\mathfrak{h}, \mathfrak{h}^{-}$, and $\mathfrak{h}^{+}$be the complexifications of $\mathfrak{h}_{0}, \mathfrak{h}_{0}^{-}$, and $\mathfrak{h}_{0}^{+}$resp. Now let $\Delta$ be the set of all roots of $\mathfrak{g}$ with respect to $\mathfrak{h}$ (simply of $\{\mathfrak{g}, \mathfrak{h}\}$ ). For every $\alpha \in A$, let $\boldsymbol{e}_{\alpha}$ be a non-zero element of $g$ such that

$$
\left[\boldsymbol{h}, \boldsymbol{e}_{\alpha}\right]=\alpha(\boldsymbol{h}) \boldsymbol{e}_{\alpha}(\boldsymbol{h} \in \mathfrak{h})
$$

and put $\mathfrak{g}_{\alpha}=\boldsymbol{C} \boldsymbol{e}_{\alpha}$ where $\boldsymbol{C}$ is the field of complex numbers. Denote by $B$ the Killing from of $\mathrm{g}$, and for every $\alpha \in \Delta$, let $\boldsymbol{h}_{\alpha}$ be a unique element of $\mathfrak{h}$ such that $B\left(\boldsymbol{h}_{\alpha}, \boldsymbol{h}\right)=\alpha(\boldsymbol{h})(\boldsymbol{h} \in \mathfrak{h})$. Put $\mathfrak{h}^{*}=\sum_{\alpha \in\lrcorner} \boldsymbol{R} \boldsymbol{h}_{\alpha}$ where $\boldsymbol{R}$ is the field of real numbers. Then $\mathfrak{h}^{*}=\mathfrak{h}_{0}^{-}+\sqrt{-1} \mathfrak{h}_{0}^{+}$and every root of $\{\mathfrak{g}, \mathfrak{h}\}$ is real valued on $\mathfrak{h}^{*}$.

Fix an ordering in the dual space of $\mathfrak{h}_{0}^{-}$and choose an ordering in the dual space of $\mathfrak{h}^{*}$ in such a way that an element $\lambda$ of the dual space of $\mathfrak{h}^{*}$ is positive whenever its restriction $\bar{\lambda}$ on $\mathfrak{h}_{0}^{-}$is positive. Let $P$ be the set of all positive roots of $\{\mathfrak{g}, \mathfrak{h}\}$ with respect to this ordering in the dual space of $\mathfrak{h}^{*}$ and let $Q$ be the set of all $\alpha \in P$ whose restrictions on $\mathfrak{h}_{0}^{-}$are not identically zero. Put $\mathfrak{n}=\sum_{\alpha \in Q} g_{\alpha}$ and $\mathfrak{n}_{0}=\mathrm{n} \cap g_{0}$. Let $N$ and $H^{-}$be the analytic subgroups of $G$ corresponding respectively to $\mathfrak{n}_{0}$ and $\mathfrak{h}_{0}^{-}$. Then $G=N H^{-} K$ is so called Iwasawa decomposition of $G$. The mapping $(n, h, u) \rightarrow n h u(n \in N$, $h \in H, u \in K)$ is an homeomorphism of $N \times H^{-} \times K$ onto $G$ [12, Ch. VI, $\S 3]$. Let $M$ be the centralizer of $\mathfrak{h}_{0}^{-}$in $K$, that is, the subgroup of $K$ consisting of all elements $u \in K$ such that $\operatorname{Ad} u(\boldsymbol{h})=\boldsymbol{h}\left(\boldsymbol{h} \in \mathfrak{h}_{0}^{-}\right)$. Then $\Gamma=N H^{-} M$ is a closed subgroup of $G$.

Now let us fix a non-zero element $\boldsymbol{h}_{0} \in \mathfrak{h}_{0}^{-}$. Let $P^{\prime}$ be the set of all $\alpha \in P$ such that $\alpha\left(\boldsymbol{h}_{0}\right)=0$. Let $\mathfrak{h}_{0}^{\prime}$ be the subalgebra of $\mathfrak{h}_{0}^{-}$ orthogonal to $P^{\prime}$, i.e., the set of all $\boldsymbol{h} \in \mathfrak{h}_{0}^{-}$fulfilling $\alpha(\boldsymbol{h})=0\left(\alpha \in P^{\prime}\right)$. Let $P^{\prime \prime}$ denote the set of all $\alpha \in P$ not identically zero on $\mathfrak{h}_{0}^{\prime}$. Then evidently $P=P^{\prime} \cup P^{\prime \prime}$. Let $S$ be the centralizer of $\mathfrak{h}_{0}^{\prime}$ in $G$. Let $\mathfrak{h}_{0}^{\prime \prime}$ be the orthogonal complement of $\mathfrak{h}_{0}^{\prime}$ in $\mathfrak{h}_{0}$ with respect to the Killing form $B$ of $g_{0}$. Put 


$$
\mathfrak{B}=\sum_{\alpha \in P \prime}\left(\mathfrak{g}_{\alpha}+\mathfrak{g}_{-\alpha}\right)+\mathfrak{h}
$$

and $\mathfrak{B}_{0}=\mathfrak{g} \cap \mathfrak{g}_{0}$. Then $\mathfrak{z}_{0}$ is the centralizer of $\mathfrak{h}_{0}^{\prime}$ in $\mathfrak{g}_{0}^{\prime}$ and the Lie algebra of $S$. It is reductive and $\mathfrak{h}_{0}=\mathfrak{h} \cap \mathfrak{g}_{0}$ is a Cartan subalgebra of it. Put $\mathfrak{g}^{\prime}=[\mathfrak{g}, \mathfrak{g}]$ and let $\mathfrak{c}_{\mathfrak{g}}$ be the center of $\mathfrak{B}$. $\mathfrak{g}^{\prime}$ is spanned over $\boldsymbol{C}$ by $\boldsymbol{h}_{\alpha}, \boldsymbol{e}_{\alpha}$, and $\boldsymbol{e}_{-\alpha}\left(\alpha \in P^{\prime}\right)$, and $c_{\bar{z}}$ is the orthogonal complement of $\left\{\boldsymbol{h}_{\alpha}, \alpha \in P^{\prime}\right\}$ in $\mathfrak{h}$ with respect to $B{ }^{2)}$ It is easily proved that $\mathfrak{B}_{0}^{\prime}=\mathfrak{S}^{\prime} \cap \mathfrak{g}_{0} \supset \mathfrak{h}_{0}^{\prime \prime}$ and $\mathfrak{c}_{\mathfrak{z}} \cap \mathfrak{g}_{0} \supset \mathfrak{h}_{0}^{\prime}$.

Let $\mathfrak{h}^{\prime \prime}$ be the complexification of $\mathfrak{h}_{0}^{\prime \prime}$ and put

$$
\mathfrak{m}^{\prime}=\mathfrak{h}^{+}+\mathfrak{h}^{\prime \prime}+\sum_{\alpha \in P^{\prime}}\left(\mathfrak{g}_{\alpha}+\mathfrak{g}_{-\alpha}\right)
$$

and $\mathfrak{m}_{0}^{\prime}=\mathfrak{m}^{\prime} \cap \mathfrak{g}_{0}$. Then $\mathfrak{m}_{0}^{\prime}$ is reductive and $\mathfrak{h}_{0}^{+}+\mathfrak{h}_{0}^{\prime \prime}$ is its Cartan subalgebra. Clearly $\mathfrak{m}_{0}^{\prime} \subset \mathfrak{S}_{0}$ and $\mathfrak{g}_{0}=\mathfrak{h}_{0}^{\prime}+\mathfrak{m}_{0}^{\prime}$. Put $\mathfrak{n}^{\prime}=\sum_{\alpha \in P^{\prime \prime}} \mathfrak{g}_{\alpha}$ and $\mathfrak{n}_{0}^{\prime}=\mathfrak{n}^{\prime} \cap \mathfrak{g}_{0}$. Then $\mathfrak{n}_{0}^{\prime}$ is nilpotent and $\left[\mathfrak{s}_{0}, \mathfrak{n}_{0}^{\prime}\right] \subset \mathfrak{n}_{0}^{\prime}$. Let $M^{\prime 0}, H^{\prime}$, and $N^{\prime}$ be the analytic subgroups corresponding respectively to $\mathfrak{m}_{0}^{\prime}, \mathfrak{h}_{0}^{\prime}$, and $\mathfrak{r}_{0}^{\prime}$. They are closed in $G$. And $N^{\prime} H^{\prime}, H^{\prime} M^{\prime 0}$, and $N^{\prime} H^{\prime} M^{\prime 0}$ are closed subgroups of $G$. Let $\Xi$ be the centralizer of $H^{\prime}$ in $K$ and put $M^{\prime}=\Xi M^{\prime 0}$. Then $S=H^{\prime} M^{\prime}$ [3(e), Corollary 3 of Lemma 26]. If $G$ is complex semisimple, $M^{\prime}$ and $S$ are connected [3(e), Lemma 27]. Put $\Gamma^{\prime}=N^{\prime} S=N^{\prime} H^{\prime} M^{\prime}$. This is a closed subgroup of $G$ and $N^{\prime}$ is normal in $\Gamma^{\prime}$. The connected component of the identity element of $S$ ( $\Gamma^{\prime}$ resp.) is $S^{0}=H^{\prime} M^{\prime 0}\left(\Gamma^{\prime 0}=N^{\prime} H^{\prime} M^{\prime 0}\right.$ resp.). Let $Z$ be the center of $G$. Then $M^{\prime} / M^{\prime 0}(D \cap Z)$ ) is finite. In fact, this is discrete and isomorphic to $\Xi / \Xi \cap M^{\prime 0}(D \cap Z)$, hence compact (because $K / D \cap Z$ is compact $[3(\mathrm{e})]$ ).

Let $S_{1}$ be a subgroup of $S$ such that $S^{0}(D \cap Z) \subset S_{1} \subset S$. Then there exists a subgroup $M_{1}$ of $M$ fulfilling $M^{\prime 0}(D \cap Z) \subset M_{1} \subset M^{\prime}$ and $S_{1}=H^{\prime} M_{1}$. Put $\Gamma_{1}=N^{\prime} S_{1}$. As $G=N H^{-} K$ and $\Gamma^{\prime 0} \supset N H^{-}$, we obtain that $G=\dot{\Gamma}_{1} K$, that is, every element $g \in G$ may be expressed as $g=\gamma u(\gamma \in \Gamma, u \in K)$. If $g$ has two expressions $g=\gamma u=\gamma^{\prime} u^{\prime}$, there exists an element $\xi \in \Xi_{1}=M_{1} \cap K$ such that $\gamma^{\prime}=\gamma \xi, u^{\prime}=\xi^{-1} u$, because $\Xi_{1}=\Gamma_{1} \cap K$.

2) A detailed proof of the facts stated here can be found in [1, pp. 199-201]. 
2. Now let us summalize some elementary results on invariant measures on groups and on homogeneous spaces. Put $\rho=2^{-1} \sum_{\alpha \in P} \alpha$ and $\rho^{\prime}=2^{-1} \sum_{\alpha \in P} \alpha$. For $g \in G$, let $\boldsymbol{h}(g)$ be a unique element of $\mathfrak{h}_{0}^{-}$such that $g \in N \exp (\boldsymbol{h}(g)) K$. Define

$$
\beta(g)=\exp \left\{\left(2 \rho-2 \rho^{\prime}\right)(\boldsymbol{h}(g))\right\} .
$$

If the element $\boldsymbol{h}_{0}$ is regular, $\rho^{\prime}$ is zero on $\mathfrak{h}_{0}^{-}$and therefore $2 \rho-2 \rho^{\prime}=2 \rho$ on $\mathfrak{h}_{0}^{-}$. Put

$$
\beta_{G}(g)=\exp \{2 \rho(\boldsymbol{h}(g))\} .
$$

As $N^{\prime}$ is normal in $\Gamma^{\prime}$, Ad $\gamma$ makes $\mathfrak{n}^{\prime}$ invariant for any $r \in \Gamma^{\prime}$. Denote by $\operatorname{Ad}_{\mathfrak{n}^{\prime}} \gamma$ the restriction of $\operatorname{Ad} \gamma$ on $\mathfrak{n}^{\prime}$, considered as a complex linear transformation.

Lemma 1. 1. For any element $\gamma \in \Gamma^{\prime}, \beta(\gamma)=\mid \operatorname{det}\left(\operatorname{Ad}_{\mathfrak{n}^{\prime}}(\gamma) \mid\right.$. If $g=\gamma \cdot u\left(\gamma \in \Gamma^{\prime}, u \in K\right)$, then $\beta(g)=\beta(\gamma)$. Moreover $\beta(\gamma g)=\beta(\gamma) \beta(g)$ $\left(r \in \Gamma^{\prime}, g \in G\right)$.

Proof. Put $\Xi=\Gamma^{\prime} \cap K=M^{\prime} \cap K . \quad \Gamma^{\prime}$ has a decomposition $\Gamma^{\prime}$ $=N H^{-} \Xi$. If $\gamma=n h \xi\left(n \in N, h \in H^{-}, \xi \in \Xi\right), \operatorname{Ad} \gamma=\operatorname{Ad} n \cdot \operatorname{Ad} h \cdot \operatorname{Ad} \xi$. For any $\boldsymbol{x} \in \mathfrak{n}, \operatorname{ad} \boldsymbol{x}$ is nilpotent on $\mathfrak{n}^{\prime}$. Therefore for any $n \in N, \operatorname{Ad} n$ is unipotent on $\mathfrak{n}^{\prime}$. Hence $\operatorname{det}\left(\operatorname{Ad}_{\mathfrak{n}^{\prime}} n\right)=1$. For any $\xi \in \Xi, \operatorname{Ad} \xi$ is a unitary transformation of $g$ with respect to the positive definite inner product $B(\boldsymbol{x}, \boldsymbol{\eta} \boldsymbol{y})(\boldsymbol{x}, \boldsymbol{y} \in \mathrm{g})$, where $\eta$ is the conjugation of $\mathrm{g}$

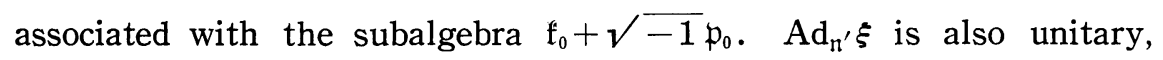
therefore $\left|\operatorname{det}\left(\operatorname{Ad}_{\mathfrak{n}^{\prime}} \xi\right)\right|=1$. Recalling that $\mathfrak{n}^{\prime}=\sum_{\alpha \in P H^{\prime}} \mathrm{g}_{\alpha}$, and $P=P^{\prime} \cup P^{\prime \prime}$, we obtain that for $h \in H^{-}$, letting $\log h \in \mathfrak{h}_{0}^{-}$be as usual,

$$
\left.\operatorname{det}\left(\operatorname{Ad}_{\mathfrak{n}^{\prime}} h\right)=\prod_{\alpha \in P^{\prime \prime}} e^{\alpha(\log h)}=\exp \left\{2 \rho-2 \rho^{\prime}\right)(\log h)\right\} .
$$

This proves the first assertion. The 2 nd is evident from the definition of $\beta$. The $3 \mathrm{rd}$ is an immediate consequence of the 1st.

Lemma 1. 2. Let $\Gamma_{1}=N^{\prime} S_{1}$ be as before.

(1) Let $d n\left(n \in N^{\prime}\right)$ and $d s\left(s \in S_{1}\right)$ be Haar measures on $N^{\prime}$ and $S_{1}$ respectively. Then $d_{r} \gamma=d n d s, d_{i \gamma}=\beta^{-1}(\gamma) d n d s$, where $\gamma=n s$ 
( $\left.n \in N^{\prime}, s \in S_{1}\right)$, are respectively a right invariant measure and a left invariant measure on $\Gamma_{1}$.

(2) $d_{r}\left(\gamma^{-1}\right)=\beta^{-1}(\gamma) d_{r} \gamma, d_{l}\left(\gamma \gamma_{0}\right)=\beta^{-1}\left(\gamma_{0}\right) d_{l \gamma} \quad\left(\gamma, \gamma_{0} \in \Gamma_{1}\right)$.

Proof. This is easy.

For $u \in K$ and $g \in G$, let $u g=n^{\prime} h^{\prime} u^{\prime}\left(n^{\prime} \in N, h^{\prime} \in H^{-}, u^{\prime} \in K\right)$. Then we put $n^{\prime}=n(u g), h^{\prime}=h(u g)$, and $u^{\prime}=u \bar{g}$. Let $u \rightarrow u^{*}(u \in K)$ be the natural mapping of $K$ onto $K^{*}=K / D \cap Z$. The coset $(u \bar{g})^{*}$ depends only on $u^{*}$, therefore it may be denoted as $u^{*} \bar{g}_{\text {. }}$. Let $C_{0}(K)$ be the set of all continuous functions on $K$ which vanish outside some compact sets. Let $d u^{*}$ be the normalized Haar measure on $K^{*}$ such that $\int_{K^{*}} d u^{*}=1$. Then there exists a Haar measure $d u$ on $K$ such that for any $f \in C_{0}(K)$,

$$
\int_{K} f(u) d u=\int_{K^{*}}\left\{\sum_{z \in D \cap z} f(z u)\right\} d u^{*} .
$$

Lemma 1. 3. Let $d n$ and $d h$ be Haar measures on $N$ and $H^{-}$ respectively.

(1) $d g=e^{-2 \rho(\log h)} d n d h d u=\beta_{G}^{-1}(g) d n d h d u$, where $g=n h u \quad(n \in N$, $\left.h \in H^{-}, u \in K\right)$, is a Haar measure on $G$.

(2) $d(u \bar{g})=\beta_{G}(u g) d u, d\left(u^{*} \bar{g}\right)=\beta_{G}(u g) d u^{*}(g \in G, u \in K)$.

Proof. This is well known [3(a), §12].

Let $\xi \rightarrow \xi^{*}$ be the natural mapping of $\Xi_{1}$ onto $\Xi_{1}^{*}=\Xi_{1} / D \cap Z$, and let $d \xi^{*}$ be the normalized Haar measure on $\Xi_{1}^{*}$ such that $\int_{\xi_{1}^{*}} d \xi^{*}=1$, and $d \xi$ be the Haar measure on $\Xi_{1}$ defined by

$$
\int_{\Xi_{1}} f(\xi) d \xi=\int_{\Xi_{1}^{*}}\left\{\sum_{z \in D \cap z} f(z \xi)\right\} d \xi^{*} \quad\left(f \in C_{0}\left(\Xi_{1}\right)\right) .
$$

There exists an invariant measure $d \tilde{u}$ on $\widetilde{K}_{1}=\Xi_{1} \backslash K=\Xi_{1}^{*} \backslash K^{*}$ which fulfills that

$$
\int_{K} f(u) d u=\int_{\tilde{K}_{1}} d \tilde{u} \int_{\Xi_{1}} f(\xi u) d \xi \quad\left(f \in C_{0}(K), \tilde{u}=\Xi_{1} u\right)
$$

and

$$
\int_{K^{*}} F\left(u^{*}\right) d u^{*}=\int_{\tilde{K}_{1}} d \tilde{u} \int_{\xi_{1}^{*}} F\left(\xi^{*} u^{*}\right) d \xi^{*} \quad\left(F \in C_{0}\left(K^{*}\right), \tilde{u}=\Xi_{1}^{*} u^{*}\right) .
$$


Let $\tilde{u} \bar{g}$ denote the element of $\widetilde{K}_{1}$ containing $u \bar{g}$, where $u$ is an representative of $\tilde{u}$. Then $\tilde{u} \rightarrow \tilde{u} \bar{g}$ is a transformation on $\widetilde{K}_{1}$.

Lemma 1. 4. $d(\tilde{u} \bar{g})=\beta(u g) d \tilde{u}\left(u \in K, g \in G, \tilde{u}=\Xi_{1} u\right)$.

Proof. As $\Gamma_{1}=N H^{-} \Xi_{1}, \Gamma_{1} \backslash G$ is isomorphic to $\widetilde{K}_{1}=\Xi_{1} \backslash K$. Let $d n$ and $d h$ be Haar measures on $N$ and $H^{-}$respectively. Then by Lemma 1. 3,

$$
d g=\beta_{G}^{-1}(h) d n d h d u \quad(g=n h u)
$$

is a Haar measure on $G$, and $d_{l \gamma}=\beta_{G}^{-1}(h) d n d h d \xi\left(\gamma=n h \xi \in \Gamma_{1}, n \in N\right.$, $\left.h \in H^{-}, \xi \in \Xi_{1}\right)$ is a left invariant measure on $\Gamma_{1}$. As is easily seen,

$$
\int_{G} f(g) d g=\int_{\tilde{K}_{1}} d \tilde{u} \int_{\Gamma_{1}} f(\gamma u) d_{i \gamma} \quad\left(f \in C_{0}(G)\right) .
$$

If $u g_{0}=\gamma^{\prime} u \bar{g}_{0} \quad(u \in K, g \in G)$, then $\beta\left(u g_{0}\right)=\beta\left(\gamma^{\prime}\right)$. Therefore using Lemma 1.2,

$$
\begin{aligned}
\int_{G} f(g) d g & =\int_{G} f\left(g g_{0}\right) d g=\int_{\widetilde{K}_{1}} d \tilde{u} \int_{\Gamma_{1}} f\left(\gamma u g_{0}\right) d r \gamma \\
& =\int_{\widetilde{K}_{1}} d \tilde{u} \int_{\Gamma_{1}} f\left(\gamma \gamma^{\prime} u \bar{g}_{0}\right) d_{l \gamma} \\
& =\int_{\tilde{K}_{1}} \beta\left(u g_{0}\right) d \tilde{u} \int_{\Gamma_{1}} f\left(\gamma u \bar{g}_{0}\right) d_{l \gamma} .
\end{aligned}
$$

Putting $\widetilde{f}(\tilde{u})=\int_{\Gamma_{1}} f(\gamma u) d_{\iota \gamma}\left(f \in C_{0}(G), u \in K\right)$, we obtain that

$$
\int_{\widetilde{K}_{1}} \widetilde{f}(\tilde{u}) d \tilde{u}=\int_{\widetilde{K}_{1}} \widetilde{f}\left(\tilde{u} \bar{g}_{0}\right) \beta\left(u g_{0}\right) d \tilde{u} .
$$

This means that $d\left(\tilde{u} \bar{g}_{0}\right)=\beta\left(u g_{0}\right) d \tilde{u}$, because the functions $\widetilde{f}$ on $\widetilde{K}_{1}\left(f \in C_{0}(G)\right)$ are sufficiently many.

Q.E.D.

As is seen from Lemmas 1.3 and 1.4 ,

$$
\int_{K^{*}} \beta_{G}(u g) d u^{*}=1, \int_{K^{*}} \beta(u g) d u^{*}=1 \quad(g \in G) .
$$

More generally we obtain the following lemma, not used in the following.

Lemma 1. 5. $\int_{\xi_{1}^{*}} \beta_{G}(\xi g) d \xi^{*}=\beta(g)(g \in G)$.

Proof. Any $f \in C_{0}\left(\widetilde{K}_{1}\right)$ may be considered as a function on $K^{*}$ 
fulfilling $f\left(\xi^{*} u^{*}\right)=f\left(u^{*}\right) \quad\left(\xi^{*} \in \Xi_{1}^{*}, u^{*} \in K^{*}\right)$. For any $g \in G$,

$$
\begin{aligned}
\int_{K^{*}} f\left(u^{*} \bar{g}\right) d u^{*} & =\int_{K^{*}} f\left(u^{*}\right) \beta_{G}\left(u g^{-1}\right) d u^{*} \\
& =\int_{\tilde{K}_{1}} f(\tilde{u})\left\{\int_{\xi_{1}^{*}} \beta_{G}\left(\xi u g^{-1}\right) d \xi^{*}\right\} d \tilde{u},
\end{aligned}
$$

where $u$ and $\xi$ are representatives of $u^{*}$ and $\xi^{*}$ respectively. On the other hand,

$$
\int_{K^{*}} f\left(u^{*} \bar{g}\right) d u^{*}=\int_{\tilde{K}_{1}} f(\tilde{u} \bar{g}) d \tilde{u}=\int_{\tilde{K}_{1}} f(\tilde{u}) \beta\left(u g^{-1}\right) d \tilde{u} .
$$

Hence $\int_{\Xi_{1}^{*}} \beta_{G}\left(\xi u g^{-1}\right) d \xi^{*}=\beta\left(u g^{-1}\right)$. This proves the lemma.

3. A representation $U$ of $G$ on a Hilbert space $E$ is a homomorphism $g \rightarrow U_{g}$ of $G$ into the group of continuous invertible operators on $E$ such that $(g, a) \rightarrow U_{g} a(g \in G, a \in E)$ is a continuous mapping of $G \times E$ into $E$. The last condition is equivalent to that $g \rightarrow U_{g} a$ is a continuous mapping of $G$ into $E$ for any $a \in E$. $U$ is said to be irreducible if there exists no closed invariant subspace different from $\{0\}$ and $E$.

Let $L$ be a representation of $S_{1}$ on a Hilbert space $E$ such that $L_{z}=\lambda(z) 1_{E}$ for all $z \in D \cap Z$, where $\lambda$ is a homomorphism of $D \cap Z$ into $C$ and $1_{E}$ is the identity operator on $E$. Let us construct canonically an induced representation $T^{L}$ of $G$ from $L$. First we study a Hilbert space $L_{2}^{L}(K)$ on which $T^{L}$ is realized. For any $u \in K$, let $\Gamma(u)$ be an element of $c_{0}$ determined uniquely by $\exp (-\Gamma(u)) u \in K^{\prime}$. There exists uniquely two real-valued linear functionals $\nu$ and $\nu^{\prime}$ on $c_{0}$ such that

$$
\lambda(z)=e^{\left(\nu+\sqrt{-1} \nu^{\prime}\right)(r(z))} \quad(z \in D \cap Z) .
$$

Hence $|\lambda(z)|=e^{\nu(r(z))}(z \in D \cap Z)$. The correspondence $\xi \rightarrow e^{-\nu(\Gamma(\xi))} L_{\xi}$ $\left(\xi \in \Xi_{1}\right)$ is a representation of $\Xi_{1}$ on $E$ and this is equivalent to a unitary one. In fact, $\xi \rightarrow e^{-\left(\nu+v-\bar{x} \nu^{\prime}\right)(r(\xi))} L_{\xi}$ is a representation of $\Xi_{1}$, which is trivial on $D \cap Z$. And hence it may be considered as a representation of the compact group $\Xi_{1}^{*}$. Therefore it is equivalent 
to a unitary representation of $\Xi_{1}^{*}$. This proves our assertion.

Let $\mathfrak{A}$ be the set of all functions $f$ with values in $E$ which fulfill the following two conditions:

(a) $f(\xi u)=L_{\xi} f(u)$ for any $u \in K$ and $\xi \in \Xi_{1}$,

(b) $f$ is (strongly) continuous.

Denote the inner product of $E$ by $(a, b)(a, b \in E)$. If $f, f_{1} \in \mathfrak{A}$, $\left(f(z u), f_{1}(z u)\right)=\left(L_{z} f(u), L_{z} f_{1}(u)\right)=e^{2 v\left(\Gamma_{(2))}\right.}\left(f(u), f_{1}(u)\right) \quad(z \in D \cap Z$, $u \in K)$. Therefore $e^{-2 v(r(u))}\left(f(u), f_{1}(u)\right)$ may be considered as a function on $K^{*}$. Introduce in $\mathfrak{A}$ a positive definite inner product

$$
\left\langle f, f_{1}\right\rangle=\int_{K^{*}}\left(f(u), f_{1}(u)\right) e^{-2 \nu(r(u))} d u^{*} .
$$

Then we obtain a Hilbert space $L_{2}^{L}(K)$ by completing $\mathfrak{A}$ with respect to the norm

$$
\|f\|=\sqrt{\langle f, f\rangle}(f \in \mathfrak{H}) .
$$

Lemma 1. 6. $L_{2}^{L}(K)$ consists of all functions $f$ on $K$ with values in $E$ fulfilling the following three conditions:

(a) $f(\xi u)=L_{\xi} f(u)$ for any $u \in K, \xi \in \Xi_{1}$,

(b') $(f(u), a)$ is measurable on $K$ for any $a \in E$,

(c) $\int_{K^{*}}(f(u), f(u)) e^{-2 \nu(r(u))} d u^{*}<+\infty$.

Of course, we must identify two functions $f_{1}$ and $f_{2}$ if $f_{1}(u)$ $=f_{2}(u)$ for almost all $u$.

First note that the integral in (c) has a sence, because the function $(f(u), f(u))$ is measurable on $K$ from $\left(\mathrm{b}^{\prime}\right)$, and

$$
(f(z u), f(z u))=e^{2 \nu(r(z))}(f(u), f(u)) \quad(z \in D \cap Z) .
$$

Let $\mu=\nu+\sqrt{-1} \nu^{\prime}$. Then as is explained above, we can define

$$
L_{\xi^{*}}^{\prime}=e^{-\mu(r(\xi))} L_{\xi}\left(\xi \in \Xi_{1}, \xi^{*}=\xi(D \cap Z)\right) .
$$

Let $\mathfrak{A}^{*}$ be the set of all functions $\varphi$ on $K^{*}$ with values in $E$ such that

(a) $\varphi\left(\xi^{*} u^{*}\right)=L_{\xi^{*}}^{\prime} \varphi\left(u^{*}\right)\left(\xi^{*} \in \Xi_{1}^{*}, u^{*} \in K^{*}\right)$, and

(B) $\varphi$ is continuous on $K^{*}$.

Let us complete $\mathfrak{A}^{*}$ with respect to the norm 


$$
\|\varphi\|=\left\{\int_{K^{*}}\left(\varphi\left(u^{*}\right), \varphi\left(u^{*}\right)\right) d u^{*}\right\}^{1 / 2} .
$$

Then we obtain a Hilbert space $L_{2}^{L^{\prime}}\left(K^{*}\right)$. The mapping $f \rightarrow \varphi\left(u^{*}\right)$ $=e^{-\mu(r(u))} f(u)$ of $\mathfrak{U}$ onto $\mathfrak{H}^{*}$ is an isomorphism, because $\left|e^{\mu(\Gamma(u))}\right|=e^{\nu(\Gamma(u))}$ ( $u \in K$ ) and hence $\|f\|=\|\varphi\|$. Therefore the above lemma is equivalent to the following one.

Lemma 1.6'. $L_{2}^{L^{\prime}}\left(K^{*}\right)$ consists of all functions on $K^{*}$ fulfilling the following three conditions: $(\alpha)$,

$\left(\beta^{\prime}\right)\left(\varphi\left(u^{*}\right), a\right)$ is measurable on $K^{*}$ for every $a \in E$, and

(r) $\int_{K^{*}}\left(\varphi\left(u^{*}\right), \varphi\left(u^{*}\right)\right) d u^{*}<+\infty$.

The norm of $\varphi$ is the square root of the above integral.

Proof. First let us remark that $\left(\varphi\left(u^{*}\right), \varphi\left(u^{*}\right)\right)$ is measurable from $\left(\beta^{\prime}\right)$. Let $\mathcal{H}$ be the Hilbert space obtained from the set of all functions $\varphi$ on $K^{*}$ with values in $E$ verifying $\left(\beta^{\prime}\right)$ and $(\gamma)$, by introducing in it the inner product

$$
\langle\varphi, \psi\rangle=\int_{K^{*}}\left(\varphi\left(u^{*}\right), \psi\left(u^{*}\right)\right) d u^{*}
$$

For any $\xi^{*} \in \Xi_{1}^{*}$, the mapping

$$
A_{\xi}^{*}: \varphi \rightarrow \varphi^{\prime}\left(u^{*}\right)=\varphi\left(\xi^{*} u^{*}\right)-L_{\xi^{*}}^{\prime} \varphi\left(u^{*}\right) \quad\left(u^{*} \in K^{*}\right)
$$

of $\mathcal{H}$ into itself is continuous. Therefore the intersection $\mathcal{H}_{1}$ of all $A_{\xi^{*}}^{-1}(0)\left(\xi^{*} \in \Xi_{1}^{*}\right)$, is a closed subspace of $\mathcal{H}$. Put

$$
(P \varphi)\left(u^{*}\right)=\int_{z_{1}^{*}} L_{\xi^{*}}^{\prime-1} \varphi\left(\xi^{*} u^{*}\right) d \xi^{*} \quad\left(\varphi \in \mathcal{H}, u^{*} \in K^{*}\right) .
$$

Then $P$ is a continuous mapping of $\mathcal{H}$ onto $\mathcal{H}_{1}$. Let $\mathfrak{B}$ be the set of all continuous functions on $K^{*}$ with values in $E$. As $\mathfrak{B}$ is dense in $\mathcal{H}, P \mathfrak{B}$ is dense in $\mathscr{H}_{1}$. On the other hand, $P \mathfrak{B}=\mathfrak{U}^{*}$. And $\mathscr{H}_{1}$ consists of all functions $\varphi$ fulfilling $\left(\alpha^{\prime}\right),\left(\beta^{\prime}\right)$, and $(\gamma)$, where $\left(\alpha^{\prime}\right)$ says that

$\left(\alpha^{\prime}\right)$ for every $\xi^{*} \in \Xi_{1}^{*}, \varphi\left(\xi^{*} u^{*}\right)=L_{\xi^{*}}^{\prime} \varphi\left(u^{*}\right)$ for almost all $u^{*}$. 
Therefore it rests only to prove that for every function $\varphi$ fulfilling $\left(\alpha^{\prime}\right),\left(\beta^{\prime}\right)$, and $(\gamma)$, there exists a function $\hat{\varphi}$ which fulfills $(\alpha),\left(\beta^{\prime}\right)$, $(\gamma)$, and $\hat{\varphi}\left(u^{*}\right)=\varphi\left(u^{*}\right)$ for almost all $u^{*}$. There exists for any such $\varphi$ a subset $A$ of $K^{*}$ such that the complement of $A$ in $K^{*}$ is of measure zero, and for any $u^{*} \in A, \varphi\left(\xi^{*} u^{*}\right)=L_{\xi^{*}}^{\prime} \varphi\left(u^{*}\right)$ for almost all $\xi^{*}$. Then $\varphi\left(\xi^{*} u^{*}\right)=L_{\xi^{*}}^{\prime} \varphi\left(u^{*}\right)$ if $u^{*} \in A$ and $\xi^{*} u^{*} \in A$. Put $\hat{\varphi}\left(\xi^{*} u^{*}\right)$ $=L_{\xi^{*}}^{\prime} \varphi\left(u^{*}\right)$ if $u^{*} \in A$ and $\xi^{*} \in \Xi_{1}^{*}$, and $\hat{\varphi}\left(u^{*}\right)=0$ if $u^{*} \notin \Xi_{1}^{*} A$. Then $\hat{\varphi}$ is well-defined and this is a function looked for.

Q.E.D.

As is suggested above, the mapping

$$
f \rightarrow \varphi\left(u^{*}\right)=e^{-\mu(\Gamma(u))} f(u)
$$

of $L_{2}^{L}(K)$ onto $L_{2}^{L \prime}\left(K^{*}\right)$ is an isomorphism.

Now let us define a representation $T^{L}$ of $G$ on $L_{2}^{L}(K)$. Extend the representation $L$ of $S_{1}$ to that of the group $\Gamma_{1}=N^{\prime} S_{1}$ by putting $L_{n}=1_{E}$ for all $n \in N^{\prime}$. For any $f \in L_{2}^{L}(K)$ and $g \in G$, put

$$
\left(T_{g}^{L} f\right)(u)=\beta^{1 / 2}(u g) L_{n\left(u_{g}\right) h(u g)} f(u \bar{g}) .
$$

As is easily seen, if $u g=\gamma u^{\prime}\left(\gamma \in \Gamma_{1}, u^{\prime} \in K\right)$,

$$
\left(T_{g}^{L} f\right)(u)=\beta^{1 / 2}(\gamma) L_{\gamma} f\left(u^{\prime}\right) .
$$

Let us prove that $g \rightarrow T_{g}^{L}(g \in G)$ defines actually a representation on $L_{2}^{L}(K)$. Denote by $f^{\prime}$ the function at the right hand side of (1.2). We see easily that $f^{\prime}$ fulfills (a) and $\left(b^{\prime}\right)$. Therefore let us check the condition (c). Denote the norm in $L_{2}^{L}(K)$ ( $E$ resp.) by $\|\cdot\|\left(\|\cdot\|_{E}\right.$ resp. $)$ and let $\zeta(u g)$ denote $n(u g) h(u g)$. Then

$$
\left\|f^{\prime}\right\|^{2}=\int_{K^{*}}\left\|L_{\zeta(u g)} f(u \bar{g})\right\|_{E}^{2} \beta(u g) e^{-2 v(r(u))} d u^{*} .
$$

The element $\zeta(u g)=n(u g) h(u g)$ depends continuously on $(u, g)$ $\in K \times G$, and may be considered as a function of $\left(u^{*}, g\right) \in K^{*} \times G$. Let $V$ be a compact subset of $G$. Then the set $\{\zeta(u g) ; u \in K, g \in V\}$ is compact in $\mathrm{NH}^{-}$. Therefore it follows from the continuity of $L$ that

$$
\sup _{u \in K, g \in V}\left|L_{\zeta(u g)}\right|=M_{V}<+\infty
$$


Similarly $e^{2 v i-r(u)+r(\overline{u g}))} \beta(u g) \beta_{G}^{-1}(u g)$ may be considered as a continuous function on $K^{*} \times G$. Therefore

$$
\sup _{u \in K, g \in V}\left\{e^{2(-r(u)+r(\bar{u} \bar{g}))} \beta(u g) \beta_{G}^{-1}(u g)\right\}=N_{v}<+\infty .
$$

Hence if $g \in V$,

$$
\begin{aligned}
\left\|f^{\prime}\right\|^{2} & \leqslant M_{V} N_{V} \int_{K^{*}}\|f(u \bar{g})\|_{E}^{2} e^{-2(\Gamma(u \bar{g}))} \beta_{G}(u g) d u^{*} \\
& =M_{V} N_{V} \int_{K^{*}}\|f(u)\|_{E}^{2} e^{-2 v(r(u))} d u^{*} \\
& =M_{V} N_{V}\|f\|^{2} .
\end{aligned}
$$

Therefore $f^{\prime} \in L_{2}^{L}(K)$, and $\left|T_{g}^{L}\right| \leqslant\left(M_{V} N_{V}\right)^{1 / 2}$ if $g \in V$.

Moreover it follows from (1.3) that $T_{s}^{L} T_{g^{\prime}}^{L}=T_{g z^{\prime}}^{L}$ for any $g, g^{\prime} \in G$ (note that $\beta(\xi)=1$ for any $\xi \in \Xi$ ).

Hence it remains only to prove the continuity of $T^{L}$. For a fixed $f \in L_{2}^{L}(K)$ and a given $\varepsilon>0$, choose an element $f_{1}$ of $\mathfrak{T}$ such that $\left\|f-f_{1}\right\|<\varepsilon$. It follows from the continuity of $L$ that for every fixed $u \in K$,

$$
\left\|T_{g}^{L} f_{1}(u)-f_{1}(u)\right\|_{E}^{2}=\left\|\beta^{1 / 2}(u g) L_{\zeta(u g)} f_{1}(u \bar{g})-f_{1}(u)\right\|_{E}^{2}
$$

tends to zero as $g \rightarrow e$.

On the other hand, let $V$ be a compact neighbourhood of $e$ in $G$, then if $g \in V$ and $u \in K$,

$$
\begin{aligned}
\| T_{g}^{L} f_{1}(u) & -f_{1}(u) \|_{E}^{2} e^{-2 v(\Gamma(u))} \\
& \leqslant\left(M_{V} N_{V}+1\right) \sup _{u \in K}\left\{\left\|f_{1}(u)\right\|_{E}^{2} e^{-2 v(\Gamma(u))}\right\}<+\infty .
\end{aligned}
$$

Because $\left\|f_{1}(u)\right\|_{E}^{2} e^{-2 \nu(r(u))}$ may be considered as a continuous function on $K^{*}$. Thus the integrand in the right hand side of

$$
\left\|T_{g}^{L} f_{1}-f_{1}\right\|^{2}=\int_{K^{*}}\left\|T_{g}^{L} f_{1}(u)-f_{1}(u)\right\|_{E}^{2} e^{-2 v(r(u))} d u^{*}
$$

is uniformly bounded for $g \in V$ and converges pointwise to zero as $g \rightarrow e$. Applying Lebesgue's theorem, we obtain that $\left\|T_{g}^{L} f_{1}-f_{1}\right\|$ $\rightarrow 0$ as $g \rightarrow e$. Hence there exists a neighbourhood $V_{\varepsilon}$ of $e$, contained in $V$, such that $\left\|T_{g}^{L} f_{1}-f_{1}\right\|<\varepsilon$ if $g \in V_{\varepsilon}$. Thus if $g \in V_{\varepsilon}$, 


$$
\left\|T_{g}^{L} f-f\right\| \leqslant\left\|T_{g}^{L}\left(f-f_{1}\right)\right\|+\left\|T_{g}^{L} f_{1}-f_{1}\right\|+\left\|f_{1}-f\right\| \leqslant \varepsilon\left(\sqrt{M_{V} N_{V}}+2\right) .
$$

This proves that $T_{g}^{L} f \rightarrow f$ as $g \rightarrow e$ and moreover $g \rightarrow T_{g}^{L} f$ is a continuous mapping of $G$ into $L_{2}^{L}(K)$. The proof of the lemma is now completed.

Remark. The representation $T^{L}$ is an induced representation of $L$ in the sence of $\left[1\right.$, p. 134] (note that $\beta(\xi)=1$ for any $\xi \in \Xi_{1}$ ).

By the isometry $f \rightarrow \varphi\left(u^{*}\right)=e^{-\mu(T(u))} f(u)$ of $L_{2}^{L}(K)$ onto $L_{2}^{L^{\prime}}\left(K^{*}\right)$, the representation $T^{L}$ in $L_{2}^{L}(K)$ is transformed into another one $U^{\mu, L}$ in $L_{2}^{L^{\prime}}\left(K^{*}\right)$. Here $U_{g}^{\mu, L}$ is defined as

$$
\begin{gathered}
\left(U_{g}^{\mu, L} \varphi\right)\left(u^{*}\right)=e^{\mu(\Gamma(u g)-\Gamma(u))} \xi^{1 / 2}(u g) L_{\zeta(u g) \varphi}\left(u^{*} \bar{g}\right) \\
\left(\varphi \in L_{2}^{L^{\prime}}\left(K^{*}\right), g \in G, u \in K\right),
\end{gathered}
$$

where $\Gamma(g)$ is a unique element of $c_{0}$ such that $g \exp (-\Gamma(g))$ $\in N H^{-} K^{\prime}$. In short, $T^{L}$ and $U^{\mu, L}$ are unitary equivalent to each other.

Lemma 1. 7. Let $L^{j}(j=1,2)$ be a representation of $S_{1}$ on a Hilbert space $E^{j}$ such that for every $z \in D \cap Z, L_{z}^{j}$ is a scalar multiple of the identity operator $1_{E^{j}}$. If $L^{1}$ and $L^{2}$ are equivalent, $T^{L^{1}}$ and $T^{L^{2}}$ are also equivalent.

Proof. Let $\nu_{j}(j=1,2)$ be the linear functional on $c_{0}$ associated with $L^{j}$. Let $A$ be an isomorphism of $E^{1}$ onto $E^{2}$ such that $L_{s}^{1}=$ $A^{-1} L_{s}^{2} A\left(s \in S_{1}\right)$. Put $|A|=a$ and $\left|A^{-1}\right|=b$. Then

$$
(a b)^{-1}\left|L_{s}^{2}\right| \leqslant\left|L_{s}^{1}\right| \leqslant a b\left|L_{s}^{2}\right| \quad\left(s \in S_{1}\right),
$$

and therefore

$$
(a b)^{-1} e^{\nu_{2}(r(z))} \leqslant e^{\nu_{1}\left(\Gamma_{(z)}\right)} \leqslant a b e^{\nu_{2}(r(z))}
$$

for any $z \in D \cap Z$. Hence $\nu_{1}=\nu_{2}$. Denote it by $\nu$.

Let $\mathcal{H}^{j}(j=1,2)$ denote $L_{2}^{L^{j}}(K)$. If $f \in \mathcal{H}^{1}$, the function $\widehat{f}(u)$ $=A(f(u))$ on $K$ with values in $E^{2}$, fulfills (a) and $\left(\mathrm{b}^{\prime}\right)$. Moreover

$$
\begin{aligned}
\|\widehat{f}\|^{2} & =\int_{K^{*}}\|\widehat{f}(u)\|_{E^{2}}^{2} e^{-2 \nu(T(u))} d u^{*} \\
& \leqslant a^{2} \int_{K^{*}}\|f(u)\|_{E^{1}}^{2} e^{-2 \nu(\Gamma(u))} d u^{*}=a^{2}\|f\|^{2} .
\end{aligned}
$$


Hence $\widehat{f} \in \mathcal{H}^{2}$, and the mapping $\widetilde{A}: f \rightarrow \widehat{f}$ of $\mathcal{H}^{1}$ into $\mathcal{H}^{2}$ is bounded and $|\widetilde{A}| \leqslant a$. Similarly we can define a continuous mapping $\left(\widetilde{A}^{-1}\right)$ of $\mathcal{H}^{2}$ into $\mathcal{H}^{1}$. Each of these operators is the inverse of the other. And moreover

$$
T_{g}^{L 1}=(\widetilde{A})^{-1} T_{g}^{L 2} \widetilde{A} \quad(g \in G) .
$$

This proves the lemma.

4. If $g \rightarrow U_{g}$ be a representation of $G$ on a Hilbert space, $g \rightarrow\left(U_{g^{-1}}\right)^{*}$ is also a representation of $G$ on the same space. This representation is denoted by $\breve{U}$.

Lemma 1. 8. If $L$ is unitary, $T^{L}$ is also unitary. In general $\left(T^{L}\right)^{\vee}$ is equivalent to $T^{\check{L}}$.

Proof. Suppcse that $L$ is not necessarily unitary. As is mentioned earlier, there exists a representation $L^{1}$ equivalent to $L$ such that $e^{-\nu(r(\xi))} L_{\xi}^{1}$ is unitary for any $\xi \in \Xi_{1}$. Therefore taking into account Lemma 1.7, we can suppose from the beginning that $e^{\left.-\nu^{\top} \Gamma(\xi)\right)} L_{\xi}$ is unitary for any $\xi \in \Xi_{1}$. If so, $\left(T^{L}\right)^{\smile}$ is unitary equivalent to $T^{\check{L}}$.

For any $f_{1}, f_{2} \in L_{2}^{L}(K)$, put $F(u)=\left(f_{1}(u), f_{2}(u)\right) \cdot e^{-2 \nu(\Gamma(u))}$. Then $F(\xi u)=F(u) \quad\left(\xi \in \Xi_{1}, u \in K\right)$, and therefore $F$ may be considered as a function on $\widetilde{K}_{1}$. Hence

$$
\begin{array}{rlrl}
\left\langle f_{1}, f_{2}\right\rangle & =\int_{K^{*}}\left(f_{1}(u), f_{2}(u)\right) e^{-2 v(r(u))} d u^{*} \quad\left(u^{*}=u(D \cap Z)\right) \\
& =\int_{\tilde{K}_{1}}\left(f_{1}(u), f_{2}(u)\right) e^{-2 v(r(u))} d \tilde{u} & \left(\tilde{u}=\Xi_{1} u\right) .
\end{array}
$$

Let us calculate $\left(T_{g^{-1}}^{L}\right) *$ for any $g \in G$.

$$
\begin{aligned}
\left\langle f_{1},\right. & \left.\left(T_{g^{-1}}^{L}\right)^{*} f_{2}\right\rangle=\left\langle T_{g^{-1}}^{L} f_{1}, f_{2}\right\rangle \\
& =\int_{\widetilde{K}_{1}}\left(\beta^{1 / 2}\left(u g^{-1}\right) L_{\zeta\left(u g^{-1}\right)} f_{1}\left(u \bar{g}^{-1}\right), f_{2}(u)\right) e^{-2 v(\Gamma(u))} d \tilde{u} \\
& =\int_{\widetilde{K}_{1}}\left(f_{1}\left(u \bar{g}^{-1}\right), L_{\zeta\left(u g^{-1}\right)}^{*} f_{2}(u)\right) \beta^{1 / 2}\left(u g^{-1}\right) e^{-2 \nu(\Gamma(u))} d \tilde{u} .
\end{aligned}
$$

Put $u \bar{g}^{-1}=v, n\left(u g^{-1}\right)=n$, and $h\left(u g^{-1}\right)=h$, then $u g^{-1}=n h v$ and hence $v g=(n h)^{-1} u$. Therefore $\zeta(v g)=(n h)^{-1}=\left(\zeta\left(u g^{-1}\right)\right)^{-1}$,

$$
\beta(v g)=\beta^{-1}(h)=\beta^{-1}\left(u g^{-1}\right), u=v \bar{g}, \tilde{u}=\hat{v} \bar{g} \text {, and } d(\hat{v} \bar{g})=\beta(v g) d \hat{v} \text {. }
$$


Hence

$\left\langle f_{1},\left(T_{g^{-1}}^{L}\right) * f_{2}\right\rangle=\int_{\tilde{K}_{1}}\left(f_{1}(v),\left(L_{\zeta(v g)}^{-1}\right) * f_{2}(v \bar{g})\right) \beta^{1 / 2}(v g) e^{-2 v(\Gamma(v(g))} d v$.

The function

$$
f^{\prime}(v)=e^{-2 \nu(\Gamma(\overline{v g})-\Gamma(v))} \beta^{1 / 2}(v g) \check{L}_{\zeta(v g)} f_{2}(v \bar{g})
$$

is an element of $L_{2}^{L}(K)$. In fact, if $v g=\gamma^{\prime} v^{\prime}\left(\gamma^{\prime} \in \Gamma_{1}, v^{\prime} \in K\right)$,

$$
e^{-2 \nu \cdot T(v \bar{v}))} \check{L}_{\zeta(v g)} f_{2}(v \bar{g})=e^{-2 v(r(v /))} \check{L_{\gamma^{\prime}}} f_{2}\left(v^{\prime}\right) .
$$

Because $\quad \Gamma_{1}=N H^{-} \Xi_{1}, \check{L_{\xi}}=\left(L_{\xi}^{-1}\right)^{*}=e^{-2 v\left(r_{(\xi)}\right)} L_{\xi}$ for any $\xi \in \Xi_{1}$, and $f_{2}(\xi u)=L_{\xi} f_{2}(u)\left(\xi \in \Xi_{1}, u \in K\right)$. Therefore $f^{\prime}(\xi v)=L_{\xi} f^{\prime}(v)$.

Thus we obtain that for $f \in L_{2}^{L}(K)$,

$$
\left(T_{g^{-1}}^{L}\right) * f(u)=e^{-2 \nu(r(\overline{\bar{g}})-r(u))} \beta^{1 / 2}(u g) \check{L}_{\zeta(u g)} f(u \bar{g}) .
$$

Consider the mapping $f \rightarrow \widehat{f}(u)=e^{-2 \nu(r(u))} f(u)$. This is an isometry of $L_{2}^{L}(K)$ onto $L_{2}^{\check{L}}(K)$. In fact

$$
\widehat{f}(\xi u)=e^{-2 \nu(r(\xi))} L_{\xi} \widehat{f}(u)=\left(\check{L}_{\xi}^{-1}\right) * \widehat{f}(u)=\check{L_{\xi}} \widehat{f}(u)
$$

and

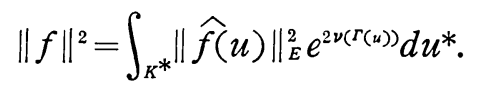

The real-valued linear functional on $\mathrm{c}_{0}$ associated with $\check{L}$ is exactly $-\nu$. Therefore the above integral is equal to the square of the norm of $f$ in $L_{2}^{\breve{L}}(K)$. By this isometry $f \rightarrow \widehat{f},\left(T_{g^{-1}}^{L}\right)^{*}$ is transformed into $T_{g^{\circ}}^{\breve{L}} \quad$ This means that $\left(T^{L}\right)^{\smile}$ is unitary equivalent to $T^{\check{L}}$.

It follows from these arguments that $T^{L}$ is unitary if $L$ is unitary. Thus the lemma is completely proved.

Remark. In this section, we fix a Cartan decompositiom $\mathfrak{g}_{0}$ $=\mathfrak{f}_{0}+\mathfrak{p}_{0}$ and a maximal abelian subalgebra $\mathfrak{h}_{0}^{-}$of $\mathfrak{p}_{0}$. But this does not restrict the generality of our discussion. In fact, the following facts are well known. (1) Let $\mathfrak{g}_{0}=\mathfrak{f}_{0}^{\prime}+\mathfrak{p}_{0}^{\prime}=\mathfrak{f}_{0}^{\prime \prime}+\mathfrak{p}_{0}^{\prime \prime}$ be two Cartan decomposition of $g_{0}$. Then there exists an element of the adjoint group $G^{*}$ of $\mathfrak{g}_{0}$ which transforms $\mathfrak{f}_{0}^{\prime}$ onto $\mathfrak{f}_{0}^{\prime \prime}$ and $\mathfrak{p}_{0}^{\prime}$ onto $\mathfrak{p}_{0}^{\prime \prime}$ resp. (2) Let $\mathfrak{g}_{0}=\mathfrak{f}_{0}+\mathfrak{p}_{0}$ be a Cartan decomposition of $\mathfrak{g}_{0}$. And let $\mathfrak{h}_{0}^{\prime}, \mathfrak{h}_{0}^{\prime \prime}$ be two 
maximal abelian subalgebras of $\mathfrak{p}_{0}$. Then there exists an element of the analytic subgroup of $G^{*}$ corresponding to $\mathfrak{t}_{0}$ which transforms $\mathfrak{G}_{0}^{\prime}$ onto $\mathfrak{G}_{0}^{\prime \prime}$. The proof of these fact is found for instance in $[7, \mathrm{pp}$. $377-381]$.

\section{$\S 2$. The existence of the characters.}

Let $A$ be a bounded operator on a separable Hilbert space $\mathscr{A}$. $A$ is called summable if for a complete orthonormal system (or simply "basis") $v_{1}, v_{2}, v_{3}, \cdots$ of $\mathcal{H}$,

$$
\sum_{i, j=1}^{\infty}\left|\left(A v_{i}, v_{j}\right)\right|<+\infty .
$$

If $A$ is summable, the condition (2.1) holds for any basis $\left\{v_{i}\right\}_{1 \leqq i<+\infty}$ of $\mathcal{H}$, and the value

$$
\sum_{i=1}^{\infty}\left(A v_{i}, v_{i}\right)
$$

does not depend on the choice of the basis of $\mathcal{H}$. This value is called the trace of $A$ and denoted by $\operatorname{Sp}(A)$. If $B_{1}, B_{2}$ be bounded operators on $\mathcal{H}, B_{1} A B_{2}$ is also summable [4(b), p. 237].

Let $G$ be a connected semisimple Lie group and $T$ a representation of $G$ on a Hilbert space $\mathcal{H}$. Let $C_{0}^{\infty}(G)$ be the set of all indefinitely differentiable functions on $G$ which vanish outside some compact sets. For any $x \in C_{0}^{\infty}(G)$,

$$
T_{x}=\int_{G} x(g) T_{g} d g
$$

is a bounded operator on $\mathcal{H}$.

Let $\Omega$ be the set of all equivalent classes of finite-dimensional irreducible representations of $K$. For $\mathscr{D} \in \Omega$, let $\mathscr{H}(\mathscr{D})$ be the set of all vectors $v \in \mathcal{H}$ such that $\left\{T_{u} v ; u \in K\right\}$ spans a finite-dimensional vector space on which $T_{u}(u \in K)$ operate as a multiple of a representation of class $\mathscr{D}$. Let $d(\mathscr{D})$ be the dimension of $\mathscr{D}$. A vector $v \in \mathscr{H}$ is called differentiable under $T$ if $f(g)=T_{g} v$ is an indefinitely (strongly) differentiable function on $G$ with values in $\mathcal{H}$. Let $\mathcal{H}^{\infty}$ denote the set of all differentiable vectors of $\mathscr{H}$. Then $\mathscr{H}^{\infty}$ is invariant 
under $T_{\boldsymbol{z}}$ for any $g \in G$, and under $T_{\boldsymbol{y}}$ for any element $\boldsymbol{y}$ of the envelopping algebra $U(\mathrm{~g})$ of $\mathrm{g}$.

$T$ is called permissible if $T_{z}$ is the scalar multiple of the unit operator on $\mathcal{H}$ for any $z \in D \cap Z . \quad T$ is called quasi-simple if it is (1) permissible and (2) $T_{z} v=\alpha(\boldsymbol{z}) v\left(\boldsymbol{z} \in \mathcal{B}, v \in \mathscr{H}^{\infty}\right)$, where 3 is the center of $U(\mathrm{~g}), \alpha$ is a homomorphism of 3 into $\boldsymbol{C}$ and is called the infinitesimal character of $T$.

Lemma 2. 1. (Harish-Chandra, [4(b) and (e)])

Let $T$ be a permissible representation of $G$ on a Hilbert space A which fulfills that

$$
\operatorname{dim} \mathscr{H}(\mathscr{D}) \leqslant N d(\mathscr{D})^{2}
$$

for every $\mathscr{D} \in \Omega$, where $N$ is a constant independent of $\mathscr{D}$. Then for any $x \in C_{0}^{\infty}(G), T_{n}$ is summable, and $x \rightarrow \mathrm{Sp}\left(T_{x}\right)$ is a distribution in the sence of L. Schwartz. If $T$ is in addition quasisimple, denoting this distribution by $\pi$,

$$
z \pi=\alpha(\boldsymbol{z}) \pi
$$

for any $z \in 3^{3)}$ And $\pi$ is essentially a locally summable function on $G$ which is analytic on the set of all regular elements of $G$.

This distribution $\pi$ is called the character of $T$.

If $T$ is cuasi-simple irreducible, it satisfies that $\operatorname{dim} \mathscr{H}(\mathscr{D})$ $\leqslant N d(\mathscr{D})^{2}(\mathscr{D} \in \Omega)$ [4(b), Lemma 4]. As is proved in Appendix, an irreducible representation $T$ is quasi-simple if and only if $\operatorname{dim} \mathscr{H}(\mathscr{D})<+\infty$ for any $\mathscr{D} \in \Omega$. If $T$ is irreducible unitary representation, it is quasi-simple [6], and therefore has the property that $\operatorname{dim} \mathcal{H}(\mathscr{D}) \leqslant N d(\mathscr{D})^{2} \quad(\mathscr{D} \in \Omega)$.

We keep to the notations of $\S 1$. Let $\omega$ be the set of all equivalent classes of irreducible finite-dimensional representations of $\Xi_{1}$. Let $d(\delta)$ be the dimension of $\delta \in \omega$. Now let $L$ be a representation

3) Every $\boldsymbol{y} \in \mathfrak{g}$ is identified with a right invariant differential operator on $G$ as $(\boldsymbol{y} x)(g)=\left.(d / d t) x(\exp (-t \boldsymbol{y}) g)\right|_{t=0} \quad\left(x \in C_{0}^{\infty}(G)\right)$.

Moreover any $y \in U(\mathfrak{g})$ may be identified with a differential operator on $G$ [9]. 
of $S_{1}$ on a Hilbert space $E$. For any $\delta \in \omega$, let $E(\delta)$ be the subspace of $E$ analogous as $\mathcal{H}(\mathscr{D})$ under $T$.

In this section, we prove the following proposition.

Proposition 1. Suppose that $L$ fulfills the following two conditions:

(1) $L_{z}=\lambda(z) 1_{E} \quad(z \in D \cap Z, \lambda(z) \in C)$,

(2) $\operatorname{dim} E(\delta) \leqslant N d(\delta)^{2} \quad(\delta \in \omega)$,

where $N$ is a constant independent of $\delta$. Then $L_{x}$ is summable for any $x \in C_{0}^{\infty}\left(S_{1}\right)$ and $L$ has a character which is a distribution on $S_{1}$. Moreover $T_{. s}^{L}$ is summable for any $x \in C_{0}^{\infty}(G)$ and $T^{L}$ has a character, a distribution on $G$.

Corsidering the uriversal covering group of $G$ if necessary, it may be assumed that $G$ is simply connected. Let us first prove the 2nd assertion.

Lemma 2.2. Suppose that $L$ fulfills the conditions (1) and (2) in the proposition. Then the induced representation $T^{L}$ is permissible, and putting $\mathcal{H}=L_{2}^{L}(K), \operatorname{dim} \mathscr{H}(\mathscr{D}) \leqslant N d(\mathscr{D})^{2}$ for every $\mathscr{D} \in \Omega$.

Proof. It is evident that $T^{L}$ is fermissible. Now take a linear functional $\mu$ on $c_{0}$ such that $L_{\xi}^{\prime}=e^{-\mu(r(\xi))} L_{\xi}\left(\xi \in \Xi_{1}\right)$ may be considered as a representation of $\Xi_{1}^{*}$. To prove the lemma, it is convenient to employ $U^{\mu, L}$ instead of $T^{L}$. Put $\mathscr{F}=L_{2}^{L \prime}\left(K^{*}\right)$. For $v \in K$ and $\varphi \in \mathscr{F}$,

$$
U_{v}^{\mu, L} \varphi\left(u^{*}\right)=e^{\mu(r(v))} \varphi\left(u^{*} v^{*}\right) .
$$

Therefore $U_{u}^{\prime}=e^{-\mu(\Gamma(u))} U_{u}^{\mu, L}(u \in K)$ may be considered as a representation of $K^{*}$ :

$$
U_{v *}^{\prime} \varphi\left(u^{*}\right)=\varphi\left(u^{*} v^{*}\right)\left(u^{*}, v^{*} \in K^{*}, \varphi \in \mathscr{F}\right) .
$$

Let $\Omega^{*}$ ( $\omega^{*}$ resp.) be the set of all equivalent classes of finite-dimensional irreducible representaticns of $K^{*}$ ( $\Xi_{1}^{*}$ resp.). If $\mathscr{E}(\mathscr{D}) \neq\{0\}$ for $\mathscr{D} \in \Omega$, taking any representation $R$ of class $\mathscr{D}, v \rightarrow e^{-\mu(\Gamma(v))} R_{v}$ $(v \in K)$ may be considered as a representation of $K^{*}$ and determines 
a class $\mathscr{D}^{*} \in \Omega^{*}$. As $d(\mathscr{D})=d\left(\mathscr{D}^{*}\right)$ and $\mathscr{L}(\mathscr{D})$ under $U^{\mu, L}$ is identical with $\mathscr{L}\left(\mathscr{D}^{*}\right)$ under $U^{\prime}$ (as vector spaces), it is safficient to verify that

$$
\operatorname{dim} \mathscr{F}\left(\mathscr{D}^{*}\right) \leqslant N d\left(\mathscr{D}^{*}\right)^{2} \quad\left(\mathscr{I}^{*} \in \Omega^{*}\right) .
$$

If $E(\delta) \neq\{0\}$ for $\delta \in \omega$, we can determine a class $\delta^{*} \in \omega^{*}$ as above. Decompose the representation $L^{\prime}$ of $\Xi_{1}^{*}$ into irreducible components. $L^{\prime}$ is equivalent to the direct sum of its restriction on $E\left(\delta^{*}\right)$ 's. $E\left(\delta^{*}\right)$ is expressed as a direct sum of irreducible components $E^{j}\left(\delta^{*}\right), 1 \leqslant j \leqslant\left(L^{\prime}: \delta^{*}\right)$, where $\left(L^{\prime}: \delta^{*}\right)$ denotes the multiplicity of $\delta^{*}$ contained in $L^{\prime}$. And $\left(L^{\prime}: \delta^{*}\right) \leqslant N d\left(\delta^{*}\right)$ from the assumption. Let us recall the general theory of induced representations of compact groups. Let $V^{j, \delta^{*}}$ be the induced representation of the restriction of $L^{\prime}$ on $E^{j}\left(\delta^{*}\right)$. Then $U^{\prime}$ is equivalent to the direct sum of

$$
V^{j, \delta^{*}} \quad\left(\delta^{*} \in \omega^{*} \text { and } 1 \leqslant j \leqslant\left(L^{\prime}: \delta^{*}\right)\right) .
$$

It is well known that $\left(V^{j, \delta^{*}}: \mathscr{D}^{*}\right)=\left(\mathscr{D}^{*}: \delta^{*}\right)$ for every $\mathscr{D}^{*} \in \Omega^{*}$. Therefore

$$
\begin{aligned}
\left(U^{\prime}: \mathscr{D}^{*}\right) & =\sum_{\delta * \in \omega *}\left(L^{\prime}: \delta^{*}\right)\left(\mathscr{D}^{*}: \delta^{*}\right) \leqslant N \sum_{\delta * \in \omega^{*}} d\left(\delta^{*}\right)\left(\mathscr{D}^{*}: \delta^{*}\right) \\
& =N d\left(\mathscr{D}^{*}\right) .
\end{aligned}
$$

Hence $\operatorname{dim} \mathscr{F}\left(\mathscr{D}^{*}\right) \leqslant N d\left(\mathscr{D}^{*}\right)^{2}$.

Q.E.D.

The 2nd assertion of Proposition 1 is an immediate consequence of Lemmas 2.1 and 2.2.

To prove the 1st assertion of the proposition, we must slitely generalise Lemma 2.1. We can prove the following lemma.

Lemma 2. 3. Let $G$ be a (not necessary connected) Lie group and $T$ a representation of $G$ on a Hilbert space $\mathcal{H}$. Suppose that there exists two closed subgroups $K$ and $D$, a continuous homomorphism $\lambda$ of $K$ into $C$, and a constant $N$, which fulfill the following conditions: (0) $D$ is normal in $K$, (1) $K / D$ is compact, (2) $T_{z}=\lambda(z) 1_{\mathcal{H}}$ for any $z \in D$, (3) $\operatorname{dim} \mathscr{H}(\mathscr{D}) \leqslant N d(\mathscr{D})^{2}$ for any equivalent class $\mathscr{D}$ of irreducible finite-dimensional representations of $K$, where $\mathcal{H}(\mathscr{D})$ 
is the set of all vectors in $\mathscr{H}$ which transform under $T_{u}(u \in K)$ according to $\mathscr{D}$. Then $T_{x}$ is summable for any $x \in C_{0}^{\infty}(G)$ and $x \rightarrow \operatorname{Sp}\left(T_{x}\right)$ is a distribution on $G$ in the sence of $L$. Schwartz.

Proof. Put $T_{u}^{*}=\lambda(u)^{-1} T_{u}$. As $T_{z u}^{*}=T_{u}^{*}(u \in K, z \in D), T^{*}$ may be considered as a representation of $K^{*}=K / D$. Let $K_{0}^{*}$ be the connected component of $K^{*}$. Then $K_{0}^{*}$ is a conneted reductive Lie group and the index of $K_{0}^{*}$ in $K^{*}$ is finite (say $n$ ). Let $\Omega^{*}\left(\Omega_{0}^{*}\right.$ resp.) be the set of all equivalent classes of irreducible finite-dimensional representations of $K^{*}\left(K_{0}^{*}\right.$ resp.). It follows from (3) that for the representation $T^{*}$ of $K^{*}$ on $\mathcal{H}, \operatorname{dim} \mathcal{H}\left(\mathscr{D}^{*}\right) \leqslant N d\left(D^{*}\right)^{2}$ for any $\mathscr{D}^{*} \in \Omega^{*}$. Consider the restriction of $T^{*}$ on $K_{0}{ }^{*}$. Then we see that $\operatorname{dim} \mathscr{H}\left(\mathscr{D}_{0}^{*}\right)$ $\leqslant n N d\left(\mathscr{D}_{0}^{*}\right)^{2}$ for any $\mathscr{D}_{0}^{*} \in \Omega_{0}^{*}$. In fact, it follows from the theory of induced representation of compact groups that any $\mathscr{D}_{0}^{*} \in \Omega_{0}^{*}$ is contained in at most $n$ different classes of $\Omega^{*}, \mathscr{L}_{1}^{*}, \mathscr{D}_{2}^{*}, \cdots, \mathscr{D}_{m}^{*}(m \leqslant n)$, and

Hence

$$
\sum_{j=1}^{m}\left[\mathscr{D}_{j}^{*}: \mathscr{D}_{0}^{*}\right] d\left(\mathscr{D}_{j}^{*}\right)=n d\left(\mathscr{D}_{0}^{*}\right) .
$$

$$
\begin{aligned}
\operatorname{dim} \mathscr{H}\left(\mathscr{D}_{0}^{*}\right) & \leqslant \sum_{j=1}^{m} \operatorname{dim} \mathscr{H}\left(\mathscr{D}_{j}^{*}\right) d\left(\mathscr{D}_{j}^{*}\right)^{-1}\left[\mathscr{D}_{j}^{*}: \mathscr{D}_{0}^{*}\right] d\left(\mathscr{D}_{0}^{*}\right) \\
& \leqslant N \sum_{j=1}^{m} d\left(\mathscr{D}_{j}^{*}\right)\left[\mathscr{D}_{j}^{*}: \mathscr{D}_{0}^{*}\right] d\left(\mathscr{D}_{0}^{*}\right)=n N d\left(\mathscr{D}_{0}^{*}\right)^{2} .
\end{aligned}
$$

Applying Lemmas 5, 6, and 7 in $[4(\mathrm{f})]$ to the restriction of $T^{*}$ on $K_{0}^{*}$, we know that Lemma 3 in $[4(\mathrm{~b})]$ is also true for it. And the proof in [4(b), pp. 241-245] of the existence of the character is valid also for $T$ without any essential modification.

Q.E.D.

Let us apply this lemma to the representation $L$ of $S_{1}$. Recall that $S_{1}$ is a subgroup of $G$ such that $S^{0}(D \cap Z) \subset S_{1} \subset S$. Therefore $\Xi^{0}(D \cap Z) \subset \Xi_{1} \subset \Xi$, where $\Xi^{0}$ is the connected component of $e$ in $\Xi$. We know that $\Xi_{1}^{*}=\Xi_{1} / D \cap Z$ is compact. For any $z \in D \cap Z, L_{z}$ $=\lambda(z) 1_{E}$ by assumption. Let $\mu$ be a linear functional on $\mathfrak{c}_{0}$ such that $e^{\mu(r(z))}=\lambda(z)(z \in D \cap Z)$. Put

$$
\lambda(\xi)=e^{\mu(r(\xi))} .\left(\xi \in \Xi_{1}\right) .
$$

Then $\lambda$ is a homomorphism of $\Xi_{1}$ into $\boldsymbol{C}$. Thus it becomes clear 
that Lemma 2.3 is applicable to the representation $L$ by taking $S_{1}$, $\Xi_{1}, D \cap Z$, and $\lambda$ respectively as $G, K, D$, and $\lambda$ in the lemma. Therefore Proposition 1 is now completely proved.

Let $G$ be a reductive Lie group which is not necessarily connected but fulfills that $G=G^{0} Z$ where $G^{0}$ is the connected component of $e$ in $G$ and $Z$ is the center of $G$. Let $g_{0}$ be the Lie algebra of $G$ and $\mathfrak{g}_{0}^{\prime}=\left[\mathfrak{g}_{0}, \mathfrak{g}_{0}\right]$. Let $\mathfrak{g}_{0}^{\prime}=\mathfrak{p}_{0}^{\prime}+\mathfrak{f}_{0}^{\prime}$ be a Cartan decomposition of the semisimple Lie algebra $\mathfrak{g}_{0}^{\prime}$, and let $K^{\prime}$ be the analytic subgroup of $G$ corresponding to $\mathfrak{f}_{0}^{\prime}$ and $K=K^{\prime} Z$. A representation $T$ of $G$ on a Hilbert space $\mathcal{H}$ is called strongly quasi-simple if it verifies the following conditions:

(1) $T_{z}=\lambda(z) 1_{\mathscr{H}} \quad(\lambda(z) \in C, z \in Z)$,

(2) $\operatorname{dim} \mathscr{H}(\mathscr{D}) \leqslant N d(\mathscr{D})^{2}$ for any equivalent class $\mathscr{D}$ of irreducible finite-dimensional representation of $K$, where $N$ is a constant independent of $\mathscr{D}$,

(3) $T_{z} v=\alpha(\boldsymbol{z}) v\left(v \in \mathcal{H}^{\infty}, \boldsymbol{z} \in \mathcal{3}\right)$, where 3 is the center of the universal envelopping algebra of the complexification $g$ of $g_{0}$.

An element $g \in G$ is called regular if the rank of $\mathrm{Ad} g$ is the maximum of those of $\operatorname{Ad} g^{\prime}\left(g^{\prime} \in G\right)$. Then we have the following lemma.

Lemma 2.4. The character of a strongly quasi-simple representation is essentially a locally summable function on $G$ which coincides with an analytic function on the set of regular elements of $G$.

This is an immediate consequence of Lemma 2.1. In $\S 5$, this is restated and applied to a representation $L$ of $S_{1}$.

In the subsequent sections, we try to express the character of $T^{L}$ by means of that of $L$. The most interesting case is the case where the character of $L$ is an invariant eigendistribution of all Laplace operators on $S_{1}$. This is treated in $\S 5$.

\section{§3. Some integral formulas on a semisimple Lie group.}

In this section, we establish some integral formulas which will 
be necessary in the later.

Let $G$ be a connected real semisimple Lie group with a Lie algebra $\mathfrak{g}_{0}$. Let $\mathfrak{h}_{0}$ be a Cartan subalgebra of $\mathfrak{g}_{0}$ and let $H$ be the Cartan subgroup of $G$ corresponding to $\mathfrak{h}_{0}$. $H$ is, by definition, the centralizer in $G$ of $\mathfrak{h}_{0}$, that is, $H \ni h$ is equivalent to $\operatorname{Ad} h(\boldsymbol{h})$ $=\boldsymbol{h}\left(\boldsymbol{h} \in \mathfrak{h}_{0}\right)$. Let $\mathfrak{g}$ and $\mathfrak{h}$ be the complexifications of $\mathfrak{g}_{0}$ and $\mathfrak{h}_{0}$ respectively. Introduce an order in the set of roots of $\{\mathfrak{g}, \mathfrak{h}\}$ and let $P$ be the set of all positive roots of $\{\mathfrak{g}, \mathfrak{h}\}$. For any root $\alpha$, let $\boldsymbol{e}_{\alpha}$ be a non-zero element of $\mathrm{g}$ such that $\left[\boldsymbol{h}, \boldsymbol{e}_{\alpha}\right]=\alpha(\boldsymbol{h}) \cdot \boldsymbol{e}_{\alpha}(\boldsymbol{h} \in \mathfrak{h})$. We see easily that for every root $\alpha$, there exists a character $\eta_{\alpha}$ of $H$ into $\boldsymbol{C}$ such that

$$
\operatorname{Ad} h\left(\boldsymbol{e}_{\alpha}\right)=\eta_{\alpha}(h) \boldsymbol{e}_{\alpha} \quad(h \in H) .
$$

We obtain $\eta_{-\alpha}(h)=\eta_{\alpha}(h)^{-1}$ from the equality $\operatorname{Ad} h\left(\left[\boldsymbol{e}_{\alpha}, \boldsymbol{e}_{-\alpha}\right]\right)=\left[\boldsymbol{e}_{\alpha}, \boldsymbol{e}_{-\alpha}\right]$.

Let $g \in G$ and consider the polynomial of $t$, $\operatorname{det}(\operatorname{Ad} g-1+t 1)$, where 1 denotes the unit operator on $g$. Let $l=\operatorname{dim}_{C} \mathfrak{h}$ and let $D(g)$ denote the cofficient of $t^{l}$, Then for any $h \in H$,

$$
\begin{aligned}
D(h) & =\prod_{\alpha \in P}\left(\eta_{\alpha}(h)-1\right)\left(\eta_{-\alpha}(h)-1\right) \\
& =\prod_{\alpha \in P} \eta_{\alpha}(h)^{-1}\left(\eta_{\alpha}(h)-1\right)^{2} .
\end{aligned}
$$

$D(g)=D\left(g_{1}\right)$, if $g=g_{0}^{-1} g_{1} g_{0}$ for some $g_{0} \in G$, and $D(g)=D\left(g^{-1}\right)$. An element $g \in G$ is called regular if $D(g) \neq 0$. Let $G^{R}$ be the set of all regular elements of $G$. Put $H^{R}=H \cap G^{R}$. For any subset $A$ of $H$, let $G_{A}$ be the set of all elements of the form $g^{-1} h g$ ( $g \in G$, $\left.h \in A \cap H^{R}\right)$.

Let $H_{0}$ be the center of $H$ and $\widetilde{H}$ the normalizer of $\mathfrak{h}_{0}$ in $G$, and put $W_{H}=\widetilde{H} / H_{0} . \quad H_{0}$ contains $Z$ and the connected component of the unit element of $H$. Hence $W_{H}$ is a finite group and this is called the Weyl group of $\{G, H\} .^{4)}$ Let $g \rightarrow \bar{g}$ be the natural mapping of $G$ onto $\bar{G}=H_{0} \backslash G$. Define $\overline{h^{g}}=g^{-1} h g$ and consider the mapping

$$
\phi:(\bar{g}, h) \rightarrow h^{\bar{g}}
$$

4) Let $A$ ( $B$ resp.) be the set of all inner automorphisms of $G$ which leave $H$ (every element of $H$ resp.) invariant. Then $W_{H}$ is canonically isomorphic to $A / B$. 
of $\bar{G} \times H^{R}$ onto $G_{H}$. As is seen in the proof of [4(e), Lemma 36, p. 488], $W_{H}$ operates on $\bar{G}$ and $H$ respectively as follows. Let $\omega \in W_{H}$ and $g_{0}$ be an element of the coset $\omega$. Then

$$
\omega \bar{g}=\left(\overline{g_{0} g}\right), h^{\omega}=g_{0} h g_{0}^{-1} \quad(g \in G, h \in H) .
$$

Hence $W_{H}$ operates on the left on $\bar{G} \times H^{R}$ as

$$
\omega(\bar{g}, h)=\left(\omega \bar{g}, h^{\omega}\right) \quad\left(\bar{g} \in \bar{G}, h \in H^{R}\right) .
$$

Now suppose that $g^{-1} h g=g^{\prime-1} h^{\prime} g^{\prime}\left(g, g^{\prime} \in G, h, h^{\prime} \in H^{R}\right)$. Then $h^{\prime}=g_{0} h g_{0}^{-1}$ where $g_{0}=g^{\prime} g^{-1}$. The sets of fixed points in $g_{0}$ of $\operatorname{Ad} h$ and $\operatorname{Ad} h^{\prime}$ are exactly $\mathfrak{h}_{0}$. Therefore $\mathfrak{h}_{0}=\operatorname{Ad} g_{0}\left(\mathfrak{h}_{0}\right)$ and hence $g_{0} \in \widetilde{H}$. Let $\omega$ be the element of $W_{H}$ containing $g_{0}$. Then $h^{\prime}=h^{\omega}$ and $\bar{g}^{\prime}=\omega \bar{g}$. Thus we see that the quotient space $W_{H} \backslash\left(\bar{G} \times H^{R}\right)$ may be identified with $G_{H}$ by the mapping $\phi$.

Before studying $\phi$ more closely, we shall make some comments. Let $g$ be an arbitrary element of $G$. Any element $\boldsymbol{x}$ of $g_{0}$ may be considered as an element of the tangent space of $G$ at $g$ as follows: for any function $f$ which is differentiable in a neighbourhood of $g$,

$$
\boldsymbol{x} f=\lim _{\boldsymbol{t} \rightarrow 0} \frac{d}{d t} f(\exp (-t \boldsymbol{x}) g),
$$

where exp denotes the exponential mapping of $\mathrm{g}_{0}$ into $G$. In this manner $\boldsymbol{x}$ defines a right invariant vector field on $G$ which is denoted by $\tilde{\boldsymbol{x}}$. Let us denote the natural mapping $g \rightarrow \bar{g}$ of $G$ onto $\bar{G}$ by $\pi$. Consider $\boldsymbol{x}$ as an element of the tangent space of $G$ at $g$, then $(d \pi)_{g} x$ is an element of the tangent space of $\bar{G}$ at $\bar{g}=\pi(g)$. Let $\psi_{g_{0}}$ be the transformation $\bar{g} \rightarrow \overline{g g} \bar{g}_{0}$ of $\bar{G}$. Then

and

$$
\left(d \psi_{g_{0}}\right)_{\bar{g}} \circ(d \pi)_{g} x=(d \pi)_{g g_{0}} x
$$

$$
(d \pi)_{h \mathbb{B}} \boldsymbol{x}=(d \pi)_{\boldsymbol{g}}\left(\operatorname{Ad}\left(h^{-1}\right) \boldsymbol{x}\right) \quad\left(h \in H_{0}\right) .
$$

The function $(d \pi)_{g} \boldsymbol{x}(g \in G)$ on $G$ may be denoted by $d \pi(\tilde{\boldsymbol{x}})$. If $\boldsymbol{x} \in \mathfrak{h}_{0}$, then $(d \pi)_{8} \boldsymbol{x}=0$.

Let $d g, d \bar{g}$, and $d h$ be invariant measures on $G, \bar{G}$, and $H$ respectively, and let $\eta, \bar{\eta}$, and $\xi$ be the right invariant differential forms 
on $G, \bar{G}$, and $H$ corresponding respectively to $d g, d \bar{g}$, and $d h$. Let $\boldsymbol{x}_{1}, \boldsymbol{x}_{2}, \cdots, \boldsymbol{x}_{r}$ be a basis of $\mathfrak{g}_{0}$ mod $\mathfrak{h}_{0}$ and $\boldsymbol{h}_{1}, \boldsymbol{h}_{2}, \cdots, \boldsymbol{h}_{l}$ be a basis of $\mathfrak{h}_{0}$. Then $(d \pi)_{g} x_{1},(d \pi)_{8} x_{2}, \cdots,(d \pi)_{8} x_{r}$ is a base of the tangent space of $\bar{G}$ at $\bar{g}$. The value $\eta_{s}\left(\boldsymbol{x}_{1}, \boldsymbol{x}_{1}, \cdots, \boldsymbol{x}_{\boldsymbol{r}}, \boldsymbol{h}_{1}, \cdots, \boldsymbol{h}_{l}\right)$ does not depend on $g$ and may be denoted by $\eta\left(\tilde{\boldsymbol{x}}_{1}, \tilde{\boldsymbol{x}}_{2}, \cdots, \tilde{\boldsymbol{x}}_{r}, \tilde{\boldsymbol{h}}_{1}, \cdots, \tilde{\boldsymbol{h}}_{l}\right)$. Since $\left(d \psi_{\varepsilon_{0}}\right)_{\bar{B}} \circ(d \pi)_{g} x=(d \pi)_{g g_{0}} x$, the value

$$
\bar{y}_{\bar{g}}\left((d \pi)_{g} x_{1}, \quad(d \pi)_{g} x_{2}, \cdots,(d \pi)_{g} x_{r}\right)
$$

does not depend on $g$ and therefore may be denoted by $\bar{\eta}\left((d \pi) \tilde{\boldsymbol{x}}_{1}\right.$, $\left.(d \pi) \tilde{\boldsymbol{x}}_{2}, \cdots,(d \pi) \tilde{\boldsymbol{x}}_{r}\right)$, Let us denote also by $\tilde{\boldsymbol{h}}_{i}$ the (right) invariant vector field on $H$ defined by $\boldsymbol{h}_{i}$ in the analogous fashion. The value $\xi_{h}\left(\boldsymbol{h}_{1}, \boldsymbol{h}_{2}, \cdots, \boldsymbol{h}_{l}\right)$ does not depend on $h$ and may be denoted by $\xi\left(\tilde{\boldsymbol{h}}_{1}, \tilde{\boldsymbol{h}}_{2}, \cdots, \tilde{\boldsymbol{h}}_{l}\right)$. Now we claim the following fact.

Lemma 3.1. Suppose that the measures $d g$, $d \bar{g}$, and $d h$ satisfy that for any $f \in C_{0}(G)$,

$$
\int_{G} f(g) d g=\int_{\bar{G}}\left\{\int_{H_{0}} f(h g) d h\right\} d \bar{g},
$$

where $\bar{g}=H_{0} g$. Then

$$
\begin{aligned}
& \eta\left(\tilde{\boldsymbol{x}}_{1}, \cdots, \tilde{\boldsymbol{x}}_{r}, \tilde{\boldsymbol{h}}_{1}, \cdots, \tilde{\boldsymbol{h}}_{l}\right) \\
& \quad=\bar{\eta}\left((d \pi) \tilde{\boldsymbol{x}}_{1}, \cdots,(d \pi) \tilde{\boldsymbol{x}}_{r}\right) \xi\left(\tilde{\boldsymbol{h}}_{1}, \cdots, \tilde{\boldsymbol{h}}_{l}\right)
\end{aligned}
$$

and vice versa.

Proof. Let $e$ be the identity element of $G$. It is sufficient for us to prove that

$$
\begin{aligned}
& \eta_{e}\left(\boldsymbol{y}_{1}, \boldsymbol{y}_{2}, \cdots, \boldsymbol{y}_{r}, \boldsymbol{z}_{1}, \boldsymbol{z}_{2}, \cdots, \boldsymbol{z}_{l}\right) \\
& \quad=\bar{\eta}_{e}^{-}\left((d \pi)_{e} \boldsymbol{y}_{1},(d \pi)_{e} \boldsymbol{y}_{2}, \cdots,(d \pi)_{e} \boldsymbol{y}_{r}\right) \xi_{e}\left(\boldsymbol{z}_{1}, \boldsymbol{z}_{2}, \cdots, \boldsymbol{z}_{l}\right)
\end{aligned}
$$

for some basis $\boldsymbol{y}_{1}, \boldsymbol{y}_{2}, \cdots, \boldsymbol{y}_{r}$ of $\mathrm{g}_{0}$ mod $\mathfrak{h}_{0}$ and some basis $\boldsymbol{z}_{1}, \boldsymbol{z}_{2}, \cdots, \boldsymbol{z}_{l}$ of $\mathfrak{h}_{0}$. We know from $[11, \S 44]$ that there exists a basis $\boldsymbol{y}_{1}, \boldsymbol{y}_{2}, \cdots$, $\boldsymbol{y}_{r}$ of $\mathfrak{g}_{0} \bmod \mathfrak{h}_{0}$, a basis $\boldsymbol{z}_{1}, \boldsymbol{z}_{2}, \cdots, \boldsymbol{z}_{l}$ of $\mathfrak{h}_{0}$, and $\varepsilon>0$ which fulfill the following conditions. Put

$$
\begin{aligned}
& g\left(t_{1}, t_{2}, \cdots, t_{l}, s_{1}, s_{2}, \cdots, s_{r}\right) \\
& \quad=\exp \left(-t_{1} \boldsymbol{z}_{1}\right) \cdots \exp \left(-t_{l} \boldsymbol{z}_{l}\right) \exp \left(-s_{1} \boldsymbol{y}_{1}\right) \exp \left(-s_{2} \boldsymbol{y}_{2}\right) \cdots \exp \left(-s_{r} \boldsymbol{y}_{r}\right) .
\end{aligned}
$$


And let $W$ be the set of all elements $g(t, s)$, where $t=\left(t_{1}, t_{2}, \cdots, t_{l}\right)$ and $s=\left(s_{1}, s_{2}, \cdots, s_{r}\right)$, such that $\left|t_{i}\right|<\varepsilon$ for $1 \leqslant i \leqslant l$ and $\left|s_{j}\right|<\varepsilon$ for $1 \leqslant j \leqslant r$. Then $W$ is a neighbourhood of $e$ in $G$ and $W \cap H_{0}$ consists of all elements $g(t, s)$ such that $\left|t_{j}\right|<\varepsilon$ for $1 \leqslant i \leqslant l$ and $s_{1}=s_{2}=\cdots=s_{r}=0$. Let $U$ be the set of all elements $g(t, s)$ such that $t_{1}=t_{2}=\cdots=t_{l}=0$ and $\left|s_{j}\right|<\varepsilon$ for $1 \leqslant j \leqslant r$. Then for every $g \in W$, there exists a unique element $u \in U$ such that $W \cap H_{0} g$ $=\left(W \cap H_{0}\right) u$. In the neighbourhood $\pi(W)=\pi(U)$ of $\bar{e}=H_{0}$ in $\bar{G}=H_{0} \backslash G$, we can take the parameter $s=\left(s_{1}, s_{2}, \cdots, s_{r}\right)$ of $u$ as the co-ordinates of $\bar{g}=H_{0} g$ with respect to some co-ordinate system of $\bar{G}$. Moreover the parameters $(t, s)$ and $t$ in $W$ and in $H_{0} \cap W$ can be taken as the co-ordinates with respect to some co-ordinate systems on $G$ and $H_{0}$ respectively.

We see easily that as the elements of the tangent space of $G$ at $e$,

$$
\boldsymbol{y}_{j}=\left(\frac{d}{d s_{j}}\right)_{0}, \boldsymbol{z}_{j}=\left(\frac{d}{d t_{i}}\right)_{0} \quad(1 \leqslant j \leqslant r, 1 \leqslant i \leqslant l),
$$

where the suffices 0 mean that the derivations are considered at $(t, s)=0$. Similarly as the elements of the tangent space of $H_{0}$ at $e$,

$$
\boldsymbol{z}_{i}=\left(\frac{d}{d t_{i}}\right)_{0} \quad(1 \leqslant i \leqslant l) .
$$

And by the definition of $d \pi$,

$$
(d \pi)_{e} \boldsymbol{y}_{j}=\left(\frac{d}{d s_{j}}\right)_{0} \quad(i \leqslant j \leqslant r)
$$

as the elements of the tangent space of $\bar{G}$ at $\bar{e}$.

Now let $d h=\sigma(t) d t_{1} d t_{2} \cdots d t_{l}(h \in W)$ and $d \bar{g}=\rho(s) d s_{1} d s_{2} \cdots d s_{r}$ ( $\bar{g} \in \pi(W)$ ), where $t$ and $s$ is the co-ordinates of $h$ and $\bar{g}$ resp., $\sigma(t)$ and $\rho(s)$ are positive continuous functions. Then it follows from (3.2) that

$$
d g=\sigma(t) \rho(s) d t_{1} d t_{2} \cdots d t_{1} d s_{1} d s_{2} \cdots d s_{r} \quad(g \in W),
$$

where $(t, s)$ is the co-ordinates of $g$. Therefore 


$$
\begin{aligned}
& \eta_{e}\left(\boldsymbol{y}_{1}, \cdots, \boldsymbol{y}_{r}, \boldsymbol{z}_{1}, \cdots, \boldsymbol{z}_{l}\right)=\sigma(0) \rho(0), \\
& \bar{\eta}_{e}\left((d \pi)_{e} \boldsymbol{y}_{1}, \cdots,(d \pi)_{e} \boldsymbol{y}_{r}\right)=\rho(0),
\end{aligned}
$$

and

$$
\xi_{c}\left(\boldsymbol{z}_{1}, \cdots, \boldsymbol{z}_{l}\right)=\sigma(0) .
$$

This proves our assertion.

Q.E.D.

Now let us assume the condition (3.2) and hence (3.3). Then reproducing the arguments of Harish-Chandra in [4(c), pp. 501-502 and p. 508], we see that

$$
\begin{aligned}
& (d \phi)_{\bar{B}, h} \circ(d \pi)_{g} \boldsymbol{x}_{i}=\operatorname{Ad}\left(g^{-1}\right)[\operatorname{Ad}(h)-1] \boldsymbol{x}_{i} \\
& (d \phi)_{\bar{g}, h} \boldsymbol{h}=\operatorname{Ad}\left(g^{-1}\right) \boldsymbol{h} \quad\left(\boldsymbol{h} \in \mathfrak{h}_{0}\right) .
\end{aligned}
$$

Therefore for $g \in G$ and $h \in H^{R}$,

$$
\begin{aligned}
& (\delta \phi \eta)_{\bar{g}, h}\left((d \pi)_{g} \boldsymbol{x}_{1}, \cdots,(d \pi)_{g} \boldsymbol{x}_{r}, \boldsymbol{h}_{1}, \cdots, \boldsymbol{h}_{l}\right) \\
& =\eta_{\bar{h} \overline{\bar{s}}}\left((d \phi)_{\bar{g}, h} \circ(d \pi)_{g} \boldsymbol{x}_{1}, \cdots,(d \phi)_{\bar{g}, h} \circ(d \pi)_{g} \boldsymbol{x}_{r},(d \phi)_{\bar{g}, h} \boldsymbol{h}_{1}, \cdots,(d \phi)_{\overline{\bar{g}}, h} \boldsymbol{h}_{l}\right) \\
& = \pm|D(h)| \eta_{h} \bar{s}\left(\boldsymbol{x}_{1}, \boldsymbol{x}_{2}, \cdots, \boldsymbol{x}_{r}, \boldsymbol{h}_{1}, \boldsymbol{h}_{2}, \cdots, \boldsymbol{h}_{l}\right) \\
& = \pm|D(h)| \bar{\gamma}_{\bar{s}}\left((d \pi)_{g} \boldsymbol{x}_{1}, \cdots,(d \pi)_{8} \boldsymbol{x}_{r}\right) \xi_{h}\left(\boldsymbol{h}_{1}, \boldsymbol{h}_{2}, \cdots, \boldsymbol{h}_{l}\right) .
\end{aligned}
$$

This proves that

$$
(\delta \phi \eta)_{\bar{g}, h}= \pm|D(h)| \zeta_{\bar{g}, h}
$$

where $\zeta$ is the differential form on $\bar{G} \times H^{R}$ corresponding to the product measure $d \bar{g} d h$.

The mapping $\phi$ is everywhere regular because $D(h) \neq 0$ for every $h \in H^{R}$. Moreover we obtain the following integral formula essentially due to Harish-Chandra.

Lemma 3.2. Let $d g, d \bar{g}$, and $d h$ be invariant measures on $G, \bar{G}$, and $H$ respectively which fulfill the condition (3.2). For any integrable function $f$ on $G_{H}$,

$$
w_{H} \int_{G_{H}} f(g) d g=\int_{\bar{G}} d \bar{g} \int_{H} f\left(h^{\bar{s}}\right)|D(h)| d h
$$

where $w_{H}$ is the order of $W_{H}$.

Let $A$ be an open subset of $H^{R}$, then $G_{A}$ is open in $G$ because 
$\phi$ is everywhere regular. We obtain from (3.4) and Lemma 3.2 the following result.

Lemma 3. 3. Let $d g, d \bar{g}$, and $d h$ be as in Lemma 3.2. Let $A$ be an open subset of $H^{R}$ and let $f$ and $\varphi$ be measurable functions on $G_{A}$ and $A$ respectively such that $f\left(h^{\bar{B}}\right) \varphi(h)$ is integrable on $\bar{G} \times A$. Then

$$
\int_{\bar{G}} \int_{A} f\left(h^{\bar{g}}\right) \varphi(h) d h d \bar{g}=\int_{G_{A}} f(g)\left\{\sum_{\omega \in W_{H}, h_{\bar{g}}^{\omega} \in A} \varphi\left(h_{g}^{\omega}\right)\right\}|D(g)|^{-1} d g,
$$

where $h_{g}$ is an element of $H^{R}$ such that $g=g_{0}^{-1} h_{g} g_{0}$ for some $g_{0} \in G$ and the summation of $\varphi\left(h_{g}^{\omega}\right)$ runs over all $\omega \in W_{H}$ such that $h_{g}^{\omega}=\left(h_{g}\right)^{\omega} \in A .^{5)}$

As an immediate generalization of Lemma 3.2, we obtain the following integral formula. Let $H^{1}, H^{2}, \cdots, H^{k}$ be a maximal set of Cartan subgroups of $G$ such that each two of them are not mutually conjugate to under any inner automorphism of $G$. Let $w_{i}$ be the order of $W_{H^{i}}$ and put $\mathcal{E}^{i}=H_{0}^{i} \backslash G$. Then for any integrable function $f$ on $G$,

$$
\int_{G} f(g) d g=\sum_{i=1}^{k} w_{i}^{-1} \int_{\varepsilon^{i}} \int_{H^{i}} f\left(h^{\bar{g}}\right)|D(h)| d h d \bar{g}
$$

where in $i$-th term, $\bar{g}=H_{0}^{i} g \in \mathcal{E}^{i}$, and $d h$ and $d \bar{g}$ are invariant measures on $H^{i}$ and $\mathcal{E}^{i}$ respectively which fulfill the analogous relations as (3.2).

Lemma 3.3 can be easily generalized in a similar manner and the generalized integral formula plays an important roll in $\S 5$.

Let $G$ be a reductive Lie group which is not necessarily connected but $G=G^{0} Z$ where $G^{0}$ and $Z$ are as in the last part of the preceding section. We can easily prove that Lemmas 3.2 and 3.3 are also valid for $G$. In this case, $H, W_{H}$, and $D(h)$ etc. may be defined analogously. In $\S 5$, we apply this generalized Lemma 3. 3 to our group $S_{1}$ which is reductive and not necessarily connected.

5) Let $h \in H$. Note that $h$ and $h^{\omega}$ may coincide for some $\omega \in W_{H}$ different from the identity element, even if $h$ is reguler. In fact, let $W^{\prime}$ be the subgroup $H / H_{0}$ of $W_{H}=\widetilde{H} / H_{0}$, then $h=h^{\omega}$ for any $\omega \in W^{\prime}$ and $h \in H_{0}$. 


\section{§. Calculation of the characters (1st step).}

In this section, we keep to the notations of $\S 1$. Let us calculate the character of the induced representation $T^{L}$. First of all, we remark the following fact. If two representations $L$ and $L^{\prime}$ of $S_{1}$ are equivalent, so are $T^{L}$ and $T^{L^{\prime}}$, and hence they have the same character. Therefore we can assume from the beginning that $e^{-\nu(\Gamma(\xi))} L_{\xi}$ is unitary for any $\xi \in \Xi_{1}$ (see $\S 1$ ).

As is proved easily, we can find a section $\mathfrak{U}$ in $K$ of $\widetilde{K}_{1}=\Xi_{1} \backslash K$ which has the following properties (1) and (2). Let $\psi$ be the mapping $u \rightarrow \tilde{u}$ of $\mathfrak{U}$ onto $\widetilde{K}_{1}$. Introduce on $\mathfrak{U}$ by the mapping $\psi$ the measure $d \mu(u)$ corresponding to the measure $d \tilde{u}$ on $\widetilde{K}_{1}$. Then

(1) the closure of $\mathfrak{U}$ in $K$ is compact,

(2) $\mathfrak{U}$ has a finite subset $U_{1}, U_{2}, \cdots, U_{p}$ such that $\mathfrak{U}-\bigcup_{i=1}^{p} U_{i}$ is of measure zero with respect to $d \mu$, each $U_{i}$ is a submanifold of $K$, and the restriction of $\psi$ on $U_{i}$ is an isomorphism of $U_{i}$ with its image in $\widetilde{K_{1}}$ as analytic manifolds.

Fix once for all a such section $\mathfrak{U}$ and put $\mathfrak{\mathfrak { U }}=\bigcup_{i=1}^{p} U_{i}$. The mapping $(\xi, u) \rightarrow \xi u$ of $\Xi \times \mathfrak{U}$ into $K$ is analytic. Conversely for $u \in K$, let $\xi(u)$ and $\phi(u)$ denote the unique elements of $\Xi_{1}$ and $\mathfrak{u}$ such that $u=\xi(u) \phi(u)$, then the mapping $u \rightarrow(\xi(u), \phi(u))$ of $K$ onto $\Xi \times \mathfrak{u}$ is analytic on every point $u$ of the set $\Xi_{1} \stackrel{\leftrightarrow}{\mathfrak{l}}$, whose complement in $K$ is of measure zero with respect to $d u$.

For any $f, f_{1} \in L_{2}^{L}(K)$,

$$
\begin{aligned}
& \left\langle f, f_{1}\right\rangle=\int_{K^{*}}\left(f(u), f_{1}(u)\right) e^{-2 \nu(\Gamma(u))} d u^{*} \\
= & \int_{K^{*}}\left(L_{\xi(u)} f(\phi(u)), L_{\xi(u)} f_{1}(\phi(u)) e^{-2,(r(\xi(u))+\Gamma(\phi(u)))} d u^{*}\right. \\
= & \int_{\mathfrak{U}}\left(f(u), f_{1}(u) e^{-2, /(r(u))} d \mu(u) .\right.
\end{aligned}
$$

Because $d u^{*}=d\left(\xi(u)^{*}\right) d \mu(\phi(u))(u \in K)$, and by assumption $e^{-\nu(r(\xi))} L_{\xi}$ is unitary for any $\xi \in \Xi_{1}$.

Let $L_{2}(\mathfrak{U})$ be the Hilbert space consisting of all square-integrable 
functions on $\mathfrak{U}$ with respect to $d \mu$. Takc; a basis, i.e, a complete orthonormal system $\left\{v_{i}\right\}_{1<i<\infty}$ of $E$, and a one $\left\{\varphi_{j}\right\}_{1 \leqslant i<\infty}$ of $L_{2}(\mathfrak{U})$. Put

$$
f_{i j}(u)=e^{\gamma(r(\phi(u)))} \varphi_{j}(\phi(u)) L_{\xi(u)} v_{i} .
$$

Lemma 4.1. The set $\left\{f_{i j}\right\}_{1 \leqslant i, j<\infty}$ is a complete orthonormal system of $L_{2}^{L}(K)$.

Proof. Using (4.1), we see that

$$
\begin{aligned}
\left\langle f_{i j}, f_{k l}\right\rangle & =\int_{\mathfrak{u}}\left(f_{i j}(u), f_{k l}(u)\right) e^{-2 v(r(u))} d \mu(u) \\
& =\int_{\mathfrak{u}} \varphi_{j}(u) \overline{\varphi_{i}(u)} d \mu(u) \cdot\left(v_{i}, v_{k}\right)=\delta_{i k} \delta_{j l} .
\end{aligned}
$$

Now suppose that $f \in L_{2}^{L}(K)$ is orthogonal to all $f_{i j}$. Since $f$ fulfills (a) in Lemma 1.6,

$$
\begin{aligned}
\left\langle f, f_{i j}\right\rangle & =\int_{K^{*}}\left(f(u), f_{i j}(u)\right) e^{-2 \nu(\Gamma(u))} d u^{*} \\
& =\int_{\mathfrak{u}}\left(f(u), v_{i}\right) e^{-\nu(\Gamma(u))} \varphi_{j}(u) d \mu(u)=0 .
\end{aligned}
$$

Therefore $\left(f(u), v_{i}\right)=0$ for any $i$ and almost all $u \in \mathfrak{U}$. Hence $f(u)=0$ for almost all $u \in \mathfrak{U}$. This means that $f=0$ as an element of $L_{2}^{L}(K)$. Thus we obtained the lemma.

Let us fix once for all a Haar measure $d g$ on $G$. Let $L$ be a representation of $S_{1}$ fulfilling the conditions (1) and (2) in Proposition 1. For any $x \in C_{0}^{\infty}(G)$, let us compute the trace of

$$
T_{x}^{L}=\int_{G} x(g) T_{g}^{L} d g
$$

Put

$$
a_{i j k}=\left\langle T_{x}^{L} f_{i j}, f_{i k}\right\rangle
$$

Then

$$
\sum_{i, j, k=1}^{\infty}\left|a_{i j k}\right|<\infty
$$

from Proposition 1. On the other hand, since $T_{x}^{L} f_{i j}$ is defined originally in the sence of convergence with respect to the norm of $L_{2}^{L}(K)$, we obtain 


$$
\left\langle T_{. j}^{L} f_{i j}, f_{i k}\right\rangle=\int_{G} x(g)\left\langle T_{g}^{L} f_{i j}, f_{i k}\right\rangle d g .
$$

And by (4.1)

$$
\begin{aligned}
a_{i j k} & =\int_{G} x(g)\left\{\int_{\mathfrak{u}}\left(T_{g}^{L} f_{i j}(u), f_{i k}(u)\right) e^{-2 v(r(u))} d \mu(u)\right\} d g \\
& =\int_{G} x(g) d g \int_{\mathfrak{u}} \beta^{1 / 2}(u g)\left(L_{\zeta(u g)} f_{i j}(u \bar{g}), f_{i k}(u)\right) e^{-2 \nu(r(u))} d \mu(u) .
\end{aligned}
$$

This double integral converges absolutely and therefore the order of the integration may be changed. Hence

$$
a_{i j k}=\int_{\mathfrak{u}} d \mu(u) \int_{G} x\left(u^{-1} g\right) \xi^{1 / 2}(g)\left(L_{\zeta(s)} f_{i j}(e \bar{g}), f_{i k}(u)\right) e^{-2 \nu(r(u)} d g .
$$

Put $g=n s v \quad\left(n \in N^{\prime}, s \in S_{1}, v \in \mathfrak{U}\right)$, then it follows from Lemma 1.2 and the 1 st equality in the proof of Lemma 1.4 that

$$
d g=\xi^{-1}(s) d n d s d \mu(v),
$$

where $d n$ and $d s$ are appropriate Haar measures on $N^{\prime}$ and $S_{1}$ respectively. Taking into account that $\beta(g)=\beta(s)$,

$$
\begin{aligned}
a_{i j k}= & \int_{\mathfrak{u} \times \mathfrak{u} \times N^{\prime} \times S_{1}} x\left(u^{-1} n s v\right)\left(L_{s} v_{i}, v_{i}\right) \\
& e^{v(r(v))} \varphi_{j}(v) e^{-\nu(\Gamma(u))} \varphi_{k}(u) \beta^{-1 / 2}(s) d n d s d \mu(v) d \mu(u) .
\end{aligned}
$$

Put

$$
\begin{aligned}
x_{j k}(s)=\int_{\mathfrak{u} \times \mathfrak{u} \times N^{\prime}} x\left(u^{-1} n s v\right) e^{\nu(\Gamma(v))} \varphi_{j}(v) \\
e^{-\nu(\Gamma(u))} \overline{\varphi_{k}(u)} d n d_{\mu}(v) d \mu(u) .
\end{aligned}
$$

Then

$$
a_{i j k}=\int_{s_{1}} x_{j k}(s) \beta^{-1 / 2}(s)\left(L_{s} v_{i}, v_{i}\right) d s .
$$

Let $\overline{\mathfrak{u}}$ be the closure of $\mathfrak{u}$ in $K$. This is compact from the condition (1) on $\mathfrak{u}$. Let $B$ be the support of $x$. Then $A=\overline{\mathfrak{u}} B \overline{\mathfrak{u}}^{-1}$ is compact and hence $A \cap \Gamma_{1}$ is compact in $\Gamma_{1}=N^{\prime} S_{1}$. The mapping $(n, s) \rightarrow n s$ of $N^{\prime} \times S_{1}$ onto $\Gamma_{1}$ is one-to-one regular everywhere. Therefore there exist compact subsets $B_{1}$ and $B_{2}$ of $N^{\prime}$ and $S_{1}$ respectively such that $\Gamma_{1} \cap A \subset B_{1} B_{2}$. If $s \notin B_{2}, x_{j k}(s)=0$. And it can be proved 
that $x_{j k}$ is indefinitely differentiable on $S_{1}$. Hence $x_{j k} \in C_{0}^{\infty}\left(S_{1}\right)$ for any $j$ and $k$. Applying the 1st part of Proposition 1 to the representation $\beta^{-1 / 2}(s) L_{s}$ of $S_{1}$ and $x_{j k} \in C_{0}^{\infty}\left(S_{1}\right)$, we obtain that for any $j$ and $k$,

$$
\sum_{i=0}^{\infty} a_{i j k}=\int_{s_{1}} x_{j k}(s) \beta^{-1 / 2}(s) \tau(s) d s,
$$

where $\tau(s)$ is the character of $L$.

Put $b_{j k}=\sum_{i=1}^{\infty} a_{i j k}$, then

$$
\operatorname{Sp}\left(T_{x}^{L}\right)=\sum_{j=1}^{\infty} b_{j j}
$$

The integral (4.4) converges to $x_{j k}$ in the sence of the ordinary convergence in $C_{0}^{\infty}\left(S_{1}\right)$ and $\beta^{-1 / 2}(s) \tau(s)$ is a distribution on $S_{1}$. Therefore

$$
b_{j k}=\int_{\mathfrak{u} \times \mathfrak{u} \times N^{\prime}} \tilde{x}(v, u, n) e^{\nu\left(r\left(v u^{-1}\right)\right)} \varphi_{j}(v) \overline{\varphi_{k}(u)} d n d \mu(v) d \mu(u),
$$

where

$$
\tilde{x}(v, u, n)=\int_{s_{1}} x\left(u^{-1} n s v\right) \beta^{-1 / 2}(s) \tau(s) d s \quad\left(v, u \in K, n \in N^{\prime}\right) .
$$

Note that $\tilde{x}$ is continuous in $(v, u, n) \in K \times K \times N^{\prime}$, and is equal to zero if $v, u \in \overline{\mathfrak{u}}$ and $n \notin B_{1}$. Therefore the above integral (4.7) converges absolutely and hence the following expression holds:

$$
b_{j k}=\int_{\mathfrak{u} \times \mathfrak{u}} K(u, v) \varphi_{j}(v) \overline{\varphi_{k}(u)} d \mu(v) d \mu(u)
$$

where

$$
K(u, v)=e^{\nu\left(r\left(v u^{-1}\right)\right)} \int_{N^{\prime}} \tilde{x}(v, u, n) d n .
$$

Now consider the integral operator $T$ on $L_{3}(\mathfrak{U})$ with the integral kernel $K(u, v)$ : for $\varphi \in L_{2}(\mathfrak{u})$,

$$
\left(T_{\varphi}\right)(u)=\int_{\mathfrak{u}} K(u, v) \varphi(v) d \mu(v) \quad(u \in \mathfrak{u}) .
$$

This operator $T$ is summable, because

$$
\sum_{j, k=1}^{\infty}\left|\left(T \varphi_{j}, \varphi_{k}\right)\right|=\sum_{j . k=1}^{\infty}\left|b_{j k}\right|<\infty
$$


where ( , ) denotes the inner product of $L_{2}(\mathfrak{l})$. Moreover

$$
\operatorname{Sp}(T)=\operatorname{Sp}\left(T_{x}^{L}\right) .
$$

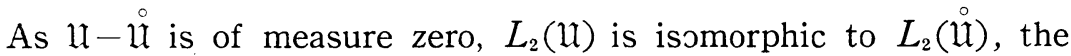
Hilbert space consisting of all square-integrable functions on $\mathfrak{U}$ with respect to the restriction of $d \mu$ on $\mathfrak{i}$. Therefore we can apply Lemma 4.2 which will be proved in the following, to the operator $T$ on $L_{2}(\mathfrak{u})$. Hence

$$
\operatorname{Sp}(T)=\int_{\mathfrak{l}} K(u, u) d \mu(u)=\int_{\mathfrak{u}} K(u, u) d \mu(u) .
$$

And therefore

$$
\operatorname{Sp}\left(T_{x}^{L}\right)=\int_{\mathfrak{u} \times N^{\prime}} \tilde{x}(u, u, n) d n d \mu(u) .
$$

If $\xi \in \Xi_{1}, n \rightarrow n^{\prime}=\xi^{-1} n \xi$ and $s \rightarrow s^{\prime}=\xi^{-1} s \xi$ are automorphism of $N^{\prime}$ and $S_{1}$ respectively and $d n^{\prime}=d n, d s^{\prime}=d s$. Moreover the distribution $\beta^{-1 / 2}(s) \tau(s)$ on $S_{1}$ is invariant under any inner automorphism of $S_{1}$. Therefore we obtain that for any $\xi \in \Xi_{1}$,

$$
\int_{N^{\prime}} \tilde{x}(u, u, n) d n=\int_{N^{\prime}} \tilde{x}(\xi u, \xi u, n) d n .
$$

Thus we proved the following theorem.

Theorem 1. Suppose $L$ is a representation of $S_{1}$ fulfilling the conditions (1) and (2) in Proposition 1. Then the characters $\tau$ and $\pi$ of $L$ and $T^{L}$ respectively exist and $\pi$ is expressed by means of $\tau$ as follows. Let $d g$, $d n$, and $d s$ be Haar measures on $G, N^{\prime}$, and $S_{1}$ respectively such that for all $x \in C_{0}(G)$,

$$
\int_{G} x(g) d g=\int_{\tilde{K}_{1}} d \tilde{u} \int_{N^{\prime} \times s_{1}} x(n s u) \beta^{-1}(s) d n d s .
$$

Then for any $x \in C_{0}^{\infty}(G)$,

$$
\begin{aligned}
& \int_{G} x(g) \pi(g) d g=\operatorname{Sp}\left(T_{x}^{L}\right) \\
& =\int_{\widetilde{K}_{1}} d \tilde{u} \int_{N^{\prime}} d n \int_{s_{1}} x\left(u^{-1} n s u\right) \beta^{-1 / 2}(s) \tau(s) d s .
\end{aligned}
$$


Now let us state Lemma 4.2. A measure space $(\mathfrak{M}, d \mu)$ is called locally euclidian (of $p$ dimensions) if it has a covering $\left\{U_{\alpha}\right\}_{\alpha \in A}$ and a one-to-one mapping $\phi_{\alpha}$ of $U_{\alpha}$ onto an open subset of $\boldsymbol{R}^{p}$ for every $\alpha \in A$, such that the transformed measure of $d \mu$ by $\phi_{\alpha}$ on $\phi_{\alpha}\left(U_{\alpha}\right)$ is absolutely continuous with respect to the usual Lebesgue measure. That is, identifying $U_{\alpha}$ with $\phi_{\alpha}\left(U_{\alpha}\right)$ by $\phi_{\alpha}$,

$$
d_{\mu}(m)=\rho_{\alpha}(x) d x_{1} d x_{2} \cdots d x_{p} \quad\left(m \in U_{\alpha}\right)
$$

where $x=\left(x_{1}, x_{2}, \cdots, x_{p}\right)=\phi_{\alpha}(m)$ and $\rho_{\alpha}$ is non negative measurable function on $\phi_{\alpha}\left(U_{\alpha}\right)$. Such family $\left\{\left(U_{\alpha}, \phi_{\alpha}\right)\right\}_{\alpha \in A}$ is called $\mu$-family temporarily. A locally euclidian measure $d \mu$ is called continucus if we can find a $\mu$-family for which any $\rho_{\alpha}$ is continuous and strictly pcsitive on $\phi_{\alpha}\left(U_{\alpha}\right)$. Suppcse that a locally euclidian measure space $(\mathfrak{M}, d \mu)$ has a $\mu$-family corsisting of only countably many $\left(U_{i}, \phi_{i}\right)$ $(i \geqslant 1)$. Then taking away frcm $\mathfrak{M}$ an appropriate subset of measure zero if necessary, we can introduce on $\mathfrak{M}$ a structure of separable $C^{0}$-manifold (or locally euclidian topological space) of $p$ dimensions such that there exists a $\mu$-family $\left\{\left(U_{i}^{\prime}, \phi_{i}^{\prime}\right)\right\}_{i 1}$ for which every $U_{i}^{\prime}$ is a co-ordinate neighbourhocd and $\phi_{i}^{\prime}$ gives the coordinates of points of $U_{i}^{\prime}$. In fact, let $U_{i}^{\prime}$ be the inverse image by $\phi_{i}$ of the set of inner points of $\phi_{i}\left(U_{i}-\bigcup_{j=1}^{i-1} U_{j}\right)$. Put $\mathfrak{M}^{\prime}=\bigcup_{i=1}^{\infty} U_{i}^{\prime}$. Then $\mathfrak{M}-\mathfrak{M}^{\prime}$ is of measure zero with respect to $d_{\mu}$. It is sufficient to introduce on $\mathfrak{M}_{i}^{\prime}$ a structure of $C^{0}$-manifold of $p$ dimensions by the family $\left\{\left(U_{i}^{\prime}, \phi_{i}^{\prime}\right)\right\}_{i \geqslant 1}$, where $\phi_{i}^{\prime}$ is the restriction of $\phi_{i}$ on $U_{i}^{\prime}$. Let $L_{2}\left(\mathfrak{M}, d_{\mu}\right)$ denote the Hilbert space of all square-integrable functions on $\mathfrak{M}$ with respect to $d_{\mu}$.

Now let $d \mu$ be a continuous locally euclidian measure on a separable $C^{0}$-manifold $\mathfrak{M}$ such that the $C^{0}$-structure is compatible with $d \mu$ in the sence above, that is, there exists a $\mu$-family $\left\{\left(U_{i}, \phi_{i}\right)\right\}_{i \geqslant 1}$ for which every $U_{i}$ is a co-ordinate neighbourhood and $\phi_{i}$ gives the co-ordinates. (All weights $\rho_{i}$ are continuous and strictly positive.) Then we have the following lemma. Denote $L_{2}(\mathfrak{M}, d \mu)$ by $\mathcal{H}$. 
Lemma 4.2. Let $T$ be a summable operator on $\mathcal{H}$. Then it is expressed by a continuous square-integrable kernel $K\left(m, m^{\prime}\right)$ as

$$
T_{\varphi}(m)=\int_{\mathfrak{M}} K\left(m, m^{\prime}\right) \varphi\left(m^{\prime}\right) d \mu\left(m^{\prime}\right) \quad(m \in \mathfrak{M}, \varphi \in \mathcal{H})
$$

and this integral converges absolutely for every $m$ and $\varphi$. Moreover the function $K(m, m)$ is integrable on $\mathfrak{M}$ and the trace of $T$ is given by

$$
\mathrm{Sp}(T)=\int_{\mathfrak{M}} K(m, m) d \mu(m) .
$$

Proof. Let $U_{j}^{\prime}$ be the set of all inner points of $U_{j}-\bigcup_{i=1}^{j-1} U_{i}$ and put $\mathfrak{M}^{\prime}=\bigcup_{j=1}^{\infty} U_{j}^{\prime}$. Then $\mathfrak{M}-\mathfrak{M}^{\prime}$ is of measure zero. Moreover for every $j$, we can find a family $\left\{O_{k}\right\}_{k \geqslant 1}$ of disjoint open subsets of $U_{j}^{\prime}$ such that (1) each $O_{k}$ is defind by

$$
a_{r}^{k}<x_{r}<b_{r}^{k} \quad(1 \leqslant r \leqslant p),
$$

where $\left(x_{1}, x_{2}, \cdots, x_{p}\right)$ is the co-ordinates in $U_{j}^{\prime}$ defined by $\phi_{j}$ and $a_{r}^{k}$, $b_{r}^{k} \in \boldsymbol{R}$, (2) denoting the clcsure of $O_{k}$ by $\bar{O}_{k}$,

$$
U_{j}^{\prime}=\bigcup_{k=1}^{\infty} \bar{O}_{k} \text {. }
$$

Assemble all such non-empty open sets over all $j$ and let them be $V_{1}, V_{2}, \cdots$ Put $\mathfrak{M}_{1}=\bigcup_{j=1}^{\infty} V_{j}$, then $\mathfrak{M}-\mathfrak{M}_{1}$ is also of measure zero. Therefore restricting $d \mu$ on $\mathfrak{M}_{1}, \mathscr{H}$ is canonically isomorphic to $L_{2}\left(\mathfrak{M}_{1}, d \mu\right)$. The latter is isomorphic to the direct sum of $\mathscr{H}_{j}=$ $L_{2}\left(V_{j}, d \mu_{j}\right)(1 \leqslant j<\infty)$, where $d \mu_{j}$ is the restriction of $d_{\mu}$ on $V_{j}$.

Consider $\mathcal{H}_{j}$ as a subspace of $\mathscr{H}$ and let $P_{j}$ be the orthogonal projection of $\mathscr{H}$ onto $\mathscr{H}_{j}$. Then $T_{j}=P_{j} T P_{j}$ is also a summable operator on $\mathscr{H}_{j}$ and

$$
\mathrm{Sp}(T)=\sum_{j=1}^{\infty} \mathrm{Sp}\left(T_{j}\right)
$$

where the sum is absolutely convergent. In fact, take a basis $\left\{\varphi_{i}^{j}\right\}_{1 \leqslant l<\infty}$ for every $\mathscr{H}_{j}$. Then

$$
\operatorname{Sp}\left(T_{j}\right)=\sum_{l=1}^{\infty}\left(T_{j} \varphi_{l}^{j}, \varphi_{l}^{j}\right)=\sum_{l=1}^{\infty}\left(T_{\varphi_{l}^{j}}^{j}, \varphi_{l}^{j}\right) .
$$


On the other hand, $\left\{\varphi_{l}^{j}\right\}_{1 \leqslant j, k \infty}$ is a basis of $\mathcal{H}$. Therefore

$$
\sum_{j, l=1}^{\infty}\left|\left(T \varphi_{l}^{j}, \varphi_{l}^{j}\right)\right|<\infty
$$

and

$$
\begin{aligned}
\operatorname{Sp}(T) & =\sum_{j, l=1}^{\infty}\left(T \varphi_{l}^{j}, \varphi_{l}^{j}\right) \\
& =\sum_{j=1}^{\infty} \sum_{l=1}^{\infty}\left(T \varphi_{l}^{j}, \varphi_{l}^{j}\right)=\sum_{j=1}^{\infty} \operatorname{Sp}\left(T_{j}\right) .
\end{aligned}
$$

Put $T_{j k}=P_{j} T P_{k}$. This is an operator of $\mathscr{H}_{k}$ into $\mathcal{H}_{j}$. Let us identify every $V_{j}$ with its image in the co-ordinate space $\boldsymbol{R}^{p}$. The following lemma is the main step of the proof of Lemma 4.2. Let $\boldsymbol{Z}^{p}$ denote the $p$-times product of the set of all integers, $\boldsymbol{Z}$.

Lemma 4. 3. For any $j$ and $k, T_{j k}$ is expressed by an continuous kernel $K_{j k}(x, y)\left(x \in V_{j}, y \in V_{k}\right)$ as

$$
T_{j k} \varphi(x)=\int_{V_{k}} K_{j k}(x, y) \varphi(y) d \mu_{k}(y) \quad\left(x \in V_{j}, \varphi \in \mathcal{H}_{k}\right) .
$$

Moreover for $j=k$,

$$
\operatorname{Sp}\left(T_{j j}\right)=\int_{V_{j}} K_{j j}(x, x) d \mu_{j}(x)
$$

and

$$
\int_{V_{j}}\left|K_{j j}(x, x)\right| d \mu_{j}(x) \leqslant \sum_{l, l^{\prime} \in z^{p}}\left|\left(T \varphi_{l}^{j}, \varphi_{l^{\prime}}^{j}\right)\right|,
$$

where $\left\{\varphi_{l}^{\prime}\right\}_{l \in z^{\triangleright}}$ is a certain basis of $\mathcal{H}_{j}$.

Proof. Suppose that $V_{j}$ is the subset of $\boldsymbol{R}^{p}$ defined by (4.14). Put for $l=\left(l_{1}, l_{2}, \cdots, l_{p}\right) \in \boldsymbol{Z}^{p}$,

$$
\varphi_{l}^{j}(x)=\frac{1}{\sqrt{\rho_{j}(x)}} \prod_{r=1}^{p} \frac{1}{\sqrt{b_{r}^{j}-a_{r}^{j}}} \exp \left\{2 \pi \sqrt{-1} l_{r} \frac{x_{r}-a_{r}^{j}}{b_{r}^{j}-a_{r}^{j}}\right\}
$$

where $\rho_{j}(x)$ is the continuous and strictly positive function defined by

$$
d \mu_{j}(x)=\rho_{j}(x) d x_{1} d x_{2} \cdots d x_{p} .
$$

Then $\left\{\varphi_{l}^{j}\right\}_{l \in z^{\triangleright}}$ is a basis of $\mathcal{H}_{j}$. Put 


$$
a_{l \prime \prime}^{j k}=\left(T_{j k} \varphi_{l,}^{k}, \varphi_{l}^{j}\right)=\left(T_{\varphi_{l}^{\prime}}^{k}, \varphi_{l}^{j}\right) .
$$

We know that $\sum_{l, l \in \in z^{p}}\left|a_{l k l}^{j k}\right|<\infty$. On the other hand,

$$
\left|\varphi_{l}^{j}(x)\right| \leqslant\left[\prod_{r=1}^{p}\left(b_{r}^{j}-a_{r}^{j}\right)\right]^{-1 / 2} \max _{x \in V_{j}} \frac{1}{\sqrt{\rho_{j}(x)}}=M_{j}<\infty .
$$

Therefore

$$
K_{j k}(x, y)=\sum_{l, l^{\prime} \in z^{\prime}} a_{l \prime \prime}^{j k} \varphi_{l}^{j}(x) \overline{\varphi_{l \prime}^{k}(y)}
$$

converges absolutely and uniformly on $V_{j} \times V_{k}$ and hence $K_{j k}$ is continuous. The expression (4.15) is evident.

If $j=k$,

$$
K_{j j}(x, x)=\sum_{l, l, \in z^{\prime}} a_{l \prime \prime}^{j,} \varphi_{l}^{j}(x) \overline{\varphi_{l \prime}^{\prime}(x)} .
$$

Therefore

$$
\int_{V_{j}} K_{j j}(x, x) d \mu_{j}(x)=\sum_{l \in z^{l}} a_{l l}^{j j}=\operatorname{Sp}\left(T_{j ;}\right)
$$

and

$$
\begin{aligned}
& \int_{V_{j}}\left|K_{j i}(x, x)\right| d \mu_{j}(x) \leqslant \sum_{1, \nu \in \boldsymbol{z}^{p}}\left|a_{l l}^{j,}\right| \int_{V_{j}}\left|\varphi_{l}^{j}(x) \overline{\varphi_{l \prime}^{j}(x)}\right| d \mu_{j}(x) \\
& \leqslant \sum_{l, l^{\prime} \in z^{p}}\left|a_{l l^{\prime}}^{j j}\right| \text {. }
\end{aligned}
$$

This proves Lemma 4. 3 .

Let us return to the proof of Lemma 4.2. Put $K^{\prime}\left(m, m^{\prime}\right)$ $=K_{j k}\left(x(m), x\left(m^{\prime}\right)\right)$ if $m \in V_{j}$ and $m^{\prime} \in V_{k^{\prime}}$ where $x(m)=\left(x_{1}(m)\right.$, $\left.x_{2}(m), \cdots, x_{p}(m)\right)$ is the co-ordinates of $m \in V_{j}$ and $x\left(m^{\prime}\right)$ is that of $m^{\prime} \in V_{k}$. Then $K^{\prime}$ is defined on $\mathfrak{M}_{1} \times \mathfrak{M}_{1}$ and continuous. Moreover for any $\varphi \in L_{2}\left(\mathfrak{M}_{1}, d \mu\right)$,

$$
T_{\varphi}(m)=\sum_{j=1}^{\infty} \int_{V_{j}} K^{\prime}\left(m, m^{\prime}\right) \varphi\left(m^{\prime}\right) d \mu\left(m^{\prime}\right),
$$

in the sence of the convergence in $L^{2}\left(\mathfrak{M}_{1}, d \mu\right)$. The function $K^{\prime}$ can be extended to a continuous function $K$ on $\mathfrak{M} \times \mathfrak{M}$. In fact, for any $m, m^{\prime} \in \mathfrak{M}$, there exists a subset $\mathfrak{M}_{1}^{\prime}$ constructed analogously as $\mathfrak{M}_{1}$ such that $m, m^{\prime} \in \mathfrak{M}_{1}^{\prime}$. Let $K^{\prime \prime}$ be the kernel defined analogously as above on $\mathfrak{M}_{1}^{\prime} \times \mathfrak{M}_{1}^{\prime}$. Then $K^{\prime}$ and $K^{\prime \prime}$ must be identical almost 
everywhere and therefore they coincide with each other on $\left(\mathfrak{M}_{1} \cap \mathfrak{M}_{1}^{\prime}\right)$ $\times\left(\mathfrak{M}_{1} \cap \mathfrak{M}_{1}^{\prime}\right)$. This proves that $K^{\prime}$ can be extended on a neighbourhood of $\left(m, m^{\prime}\right)$ as a continuous function. The extended function $K$ on $\mathfrak{M}$ of $K^{\prime}$ is the kernel looked for. $K$ is square-integrable because

$$
\begin{gathered}
\int_{\mathfrak{M} \times \mathfrak{M}}\left|K\left(m, m^{\prime}\right)\right|^{2} d \mu(m) d \mu\left(m^{\prime}\right)=\sum_{j, k=1}^{\infty} \int_{V_{j} \times V_{k}}\left|K_{j k}(x, y)\right|^{2} d \mu_{j}(x) d \mu_{k}(y) \\
=\sum_{j, k=1}^{\infty} \sum_{l, l, \in z^{\rho}}\left|a_{l \prime \prime}^{j k}\right|^{2}<\infty .
\end{gathered}
$$

Moreover for any $m \in \mathfrak{M}$,

$$
\int_{\mathfrak{M}}\left|K\left(m, m^{\prime}\right)\right|^{2} d \mu\left(m^{\prime}\right)<+\infty .
$$

In fact, we can assume that $m \in V_{j}$ for some $j$. Then

$$
\begin{aligned}
& \int_{\mathfrak{M}}\left|K\left(m, m^{\prime}\right)\right|^{2} d \mu\left(m^{\prime}\right)=\sum_{k=1}^{\infty} \int_{v_{k}}\left|K_{j k}\left(m, m^{\prime}\right)\right|^{2} d \mu\left(m^{\prime}\right) \\
& =\sum_{k=1}^{\infty} \sum_{l, l, l l^{\prime \prime} \in z^{\ell}} a_{l \prime \prime \prime}^{j k} \overline{l_{l \prime \prime \prime \prime}^{\prime k}} \varphi_{l}^{j}(m) \overline{\varphi_{l \prime}^{j}(m)} \\
& \leqslant M_{j}^{2} \sum_{k=1}^{\infty} \sum_{l, l,, l \prime \prime \in z^{\ell}}\left|a_{l \prime \prime \prime}^{j k} a_{l \prime \prime \prime \prime \prime}^{j k}\right|<+\infty .
\end{aligned}
$$

From (4.16), we see that the integral (4.13) converges absolutely for any $m \in \mathfrak{M}$ and $\varphi \in \mathcal{H}$.

Now

$$
\begin{gathered}
\int_{\mathfrak{M}}|K(m, m)| d \mu(m)=\sum_{j=1}^{\infty} \int_{v !}\left|K_{j j}(x, x)\right| d \mu_{j}(x) \\
\leqslant \sum_{j=1}^{\infty} \sum_{l, l^{\prime \prime} \in \boldsymbol{z}^{\dagger}}\left|a_{l l^{\prime}}^{j j}\right|<+\infty .
\end{gathered}
$$

Therefore the following integral is absolutely convergent.

$$
\begin{aligned}
\int_{\mathfrak{R}} K(m, m) d \mu(m) & =\sum_{j=1}^{\infty} \int_{v_{j}} K_{j j}(x, x) d \mu_{j}(x) \\
= & \sum_{j=1}^{\infty} \operatorname{Sp}\left(T_{j}\right) .
\end{aligned}
$$

Thus we now proved that

$$
\operatorname{Sp}(T)=\int_{\mathfrak{M}} K(m, m) d \mu(m) .
$$


Remark. An operator $T$ on a Hilbert space $E$ is called of Hilbert-Schmidt type if

$$
\sum_{j=1}^{\infty}\left\|T e_{j}\right\|^{2}<+\infty
$$

for a basis $\left\{e_{j}\right\}_{1<j<\infty}$ of $E$, where $\|\cdot\|$ denotes the norm of $E$. As is well known, the product of any two operators of Hilbert-Schmidt type, is summable.

\section{$\S 5$. Calculation of the characters (2nd step).}

To obtain a more explicite formula for the character of $T^{L}$, we must assume that $S_{1}$ and its representation $L$ fulfill more stronger conditions.

As the readers may have been noticed, the assumption in $\S \S 1,2$, and 4 that $G$ is simply connected is artificial. This one is made only to define well the functions $\Gamma(g)$ on $G, \nu, \nu^{\prime}$, and $\mu$ on $\mathfrak{c}_{0}$. Here we cast off this assumption as follows. Let $Z_{1}$ be the subgroup of $Z \cap S_{1}$ consisting of all elements $z$ such that $L_{z}=1_{E}$. Then as is easily seen, $T_{z}^{L}=1_{\mathscr{H}}$ for every $z \in Z_{1}$, where $\mathscr{H}$ denotes $L_{2}^{L}(K)$. Therefore $L$ and $T^{L}$ may be considered as representations of the factor groups $S_{1} / Z_{1}$ and $G / Z_{1}$ respectively. Accordingly, their characters $\tau$ and $\pi$ may be considered as the distributions on these factor groups respectively. For our pourpose, there exists no essential difference for considering $G / Z_{1}$ and $S_{1} / Z_{1}$ instead of $G$ and $S_{1}$. Hence in this section, we denote the factor groups $G / Z_{1}$ and $S_{1} / Z_{1}$ again by $G$ and $S_{1}$ respectively. Moreover we denominate another notations as well, for instance, $S / Z_{1}$ is denoted again by $S$, and the notations $L, T^{L}, \tau$, and $\pi$ are preserved. Then Theorem 1 in the preceding section remains valid for this case.

Let $Z_{S_{1}}$ be the center of $S_{1}$ and $Z_{3}$ the center of the universal envelopping algebra of $\boldsymbol{z}$. In this section, we assume that (i) $S_{1}=S^{0} Z_{S_{1}}$ and $S^{0}(D \cap Z) \subset S_{1} \subset S$, and (ii) $L$ is a strongly quasi-simple representation. By definition, $L$ is strongly quasi-simple if it verifies that
(1)
$L_{z}=\lambda(z) 1_{E}$
$\left(\lambda(z) \in \boldsymbol{C}, z \in Z_{s_{1}}\right)$, 
(2) $\operatorname{dim} E(\delta) \leqslant N d(\delta)^{2}(\delta \in \omega)$, where $N$ is a constant independent of $\delta$,

(3) $L_{z} a=\alpha(z) a \quad\left(\alpha(z) \in C, a \in E^{\infty}, z \in \beta_{3}\right)$.

Then as is easily proved, the character $\tau$ of $L$ fulfills that

$$
\boldsymbol{z} \tau=\alpha(\boldsymbol{z}) \tau \quad(\boldsymbol{z} \in \mathcal{B}) .
$$

These conditions on $S_{1}$ and $L$ are stronger than the ones (1) and (2) in Proposition 1.

Lemma 5. 1. Under these assumptions on $S_{1}$ and $L, \tau(s)$ is essentially a locally summable function on $S_{1}$ and the multiple integral in the right hand side of

$$
\operatorname{Sp}\left(T_{x}^{L}\right)=\int_{\tilde{\kappa}_{1}} d \tilde{u} \int_{N^{\prime}} d n \int_{s_{1}} x\left(u^{-1} n s u\right) \beta^{-1 / 2}(s) \tau(s) d s \quad\left(\tilde{u}=\Xi_{1} u\right)
$$

is absolutely convergent.

Proof. Let $\mathfrak{h}_{0}$ and $\mathfrak{h}_{0}^{\prime}$ be as in $\S 1$. Put $l=\operatorname{dim} \mathfrak{h}_{0}$ and $l^{\prime}=\operatorname{dim} \mathfrak{h}_{0}^{\prime}$. Let $A d_{\mathfrak{s}} s$ denote the restriction on $\mathfrak{s}$ of $A d s$ on g. And let $D_{\mathfrak{\xi}}(s)$ be the coefficient of $t^{t-l \prime}$ of $\operatorname{det}\left(A d s-1_{\mathfrak{s}}+t 1_{\mathfrak{z}}\right)$, where $1_{\mathfrak{z}}$ is the unit operator on 3 . Then $D_{\mathfrak{3}}(s)=D_{\mathfrak{3}}(m)$ if $s=h m \quad\left(h \in H^{\prime}, m \in M^{\prime}\right)$. We know the following two facts due to Harish-Chandra: (1) the function $\left|D_{\mathfrak{S}}(s)\right|^{-1 / 2}$ is integrable on any compact subset of $S_{1}$ [4(e), p. 504], (2) $\left|D_{\mathfrak{Z}}(s)\right|^{1 / 2} \tau(s)$ is a function defined at least on the set of all regular elements of $S_{1}$, which is bounded on every compact subset of $S_{1}\left[4(\mathrm{~d})^{6}\right)$ pp. 129-134, and 4(e), p. 477]. From these two facts, the assertions of the lemma follow immediately.

Now let $\mathfrak{y}_{0}, \mathfrak{h}_{0}^{\prime}, \mathfrak{h}_{0}^{+}$, and $\mathfrak{h}_{0}^{-}$be as in $\S 1 . \mathfrak{h}_{0}=\mathfrak{h}_{0}^{-}+\mathfrak{h}_{0}^{+}$is a Cartan subalgebra of $\mathfrak{g}_{0}$. Let $\mathfrak{h}_{0}^{1}=\mathfrak{h}_{0}, \mathfrak{h}_{0}^{2}, \cdots, \mathfrak{h}_{0}^{k}$ be a maximal set of Cartan subalgebras of $\mathfrak{g}_{0}$ such that each two of which are not conjugate to one another under any inner automorphism of $S_{1}$. Every $\mathfrak{h}_{0}^{j}$ contains $\mathfrak{h}_{0}^{\prime}$, and is also a Cartan subalgebra of $\mathfrak{g}_{0}$ because $\operatorname{dim} \mathfrak{h}_{0}^{j}=\operatorname{dim} \mathfrak{h}_{0}$.

6) Note that the correct definition of Cartan subgroups of a semisimple Lie group is found in p. 556 of "Harish-Chandra, Some results on an invariant integral on a semisimple Lie algebra, Ann. Math., 80 (1964), pp. 551-593," 
(We can choose every $\mathfrak{h}_{0}^{j}$ in such a manner that $\mathfrak{h}_{0}^{j}=\mathfrak{h}_{0}^{j} \cap \mathfrak{f}_{0}+\mathfrak{h}_{0}^{j} \cap \mathfrak{p}_{0}$ and $\mathfrak{h}_{0}^{j} \cap \mathfrak{p}_{0} \subset \mathfrak{h}_{0}^{-}[7$, p. 383]).

Let $A^{j}$ ( $H^{j}$ resp.) be the Cartan subgroup of $S_{1}$ ( $G$ resp.) corresponding to $\mathfrak{h}_{0}^{j}$. Then clearly $H^{j} \cap S_{1}=A^{j}$. Moreover $H^{j} \subset S$ because any $h \in H^{j}$ must commutes with all elements of $\mathfrak{h}_{0}^{\prime}$. Let $A_{0}^{j}\left(H_{0}^{j}\right.$ resp.) be the center of $A^{j}$ ( $H^{j}$ resp.) Then $H_{0}^{j} \cap S_{1}=H_{0}^{j} \cap A^{j}=H_{0}^{j} \cap A_{0}^{j}$. Put $Z^{j}=H_{0}^{j} \cap A_{0}^{j}$. Since $Z^{j}$ contains the connected component of the identity element $e$ in $H$ and $Z \cap D$, the indices $p_{j}=\left[H_{0}^{j}: Z^{j}\right]$ and $q_{j}=\left[A_{0}^{j}: Z^{j}\right]$ are finite, where $\left[H_{0}^{j}: Z^{j}\right]$ denotes the number of the elements of $H_{0}^{j} / Z^{j}{ }^{\text {. }}$ ) If $G$ is complex semisimple, $S$ and $H^{j}$ 's are connected [4(e), p. 482], and moreover $k=1$. Therefore $S_{1}=S$ and $A^{1}=A_{0}^{1}=H^{1}=H_{0}^{1}$, hence $p_{1}=q_{1}=1$. Let $S_{A^{j}}$ be the set of all elements of $S_{1}$ which can be expressed as $s^{-1} h s\left(s \in S_{1}, h \in A^{j} \cap G^{R}\right)$. Then $S_{A^{i}} \cap S_{A^{j}}=\phi$ if $i \neq j$, and $S_{1} \cap G^{R}=\bigcup_{j=1}^{k} S_{A^{j}}$. And for any $g \in G, g^{-1} S_{A^{j}} g$ $\subset G_{H^{j}}$.

For every $j(1 \leqslant j \leqslant k)$, let $d^{j} h$ be a Haar measure on $H^{j}$ and let $d^{j} \widehat{\mathcal{S}}$ be a Haar measure on $\widehat{S}^{j}=A_{0}^{j} \backslash S_{1}$ such that

$$
\int_{S_{1}} \varphi(s) d s=\int_{\widehat{S}^{j}} d^{j} \hat{s} \int_{A_{i}^{j}} \varphi(h s) d^{j} h \quad\left(\varphi \in C_{0}\left(S_{1}\right)\right),
$$

where $\hat{s}=A_{0}^{j} s$. Apply (3.5) to every term of the right hand side of

$$
\int_{s_{1}} x\left(u^{-1} n s u\right) \beta^{-1 / 2}(s) \tau(s) d s=\sum_{j=1}^{k} \int_{s_{A^{j}}} x\left(u^{-1} n s u\right) \beta^{-1 / 2}(s) \tau(s) d s .
$$

Then (5.1) becomes

(5.3) $\quad \operatorname{Sp}\left(T_{x}^{L}\right)$

$$
=\sum_{j=1}^{k} \frac{1}{w_{j}} \int_{\widetilde{K}_{1}} d \tilde{u} \int_{N^{\prime}} d n \int_{\widehat{S}^{j}} d^{j} \hat{s} \int_{A^{j}} x\left(u^{-1} n s^{-1} h s u\right) \beta^{-1 / 2}(h) \tau(h)\left|D_{\mathfrak{\xi}}(h)\right| d^{j} h,
$$

where $w_{j}$ is the order of the Weyl group $W_{A^{j}}$ of $\left\{S_{1}, A^{j}\right\}$, becouse

$$
\beta^{-1 / 2}\left(s^{-1} h s\right) \tau\left(s^{-1} h s\right)=\beta^{-1 / 2}(h) \tau(h) .
$$

To rewrite (5.3), we need the following lemma.

7) See $[4(\mathrm{e})$, p. 481$]$ and recall that $K / D \cap Z$ is compact. 
Lemma 5.2. If $s \in S \cap G^{R}$, the mapping $n \rightarrow n^{\prime} \equiv n^{-1} s n s^{-1}$ $\left(n \in N^{\prime}\right)$ is one-to-one regular mapping of $N^{\prime}$ onto itself. And

$$
\begin{aligned}
d n^{\prime} & =\left|\operatorname{det}\left(\operatorname{Ad}_{\mathfrak{n}^{\prime}} s-1_{\mathfrak{n}^{\prime}}\right)\right| d n \\
& =|D(s)|^{1 / 2}\left|D_{\mathfrak{\xi}}(s)\right|^{-1 / 2} \beta^{1 / 2}(s) d n,
\end{aligned}
$$

where $\operatorname{Ad}_{\mathfrak{n}^{\prime}} s$ denotes the restriction of $\operatorname{Ad} s$ on $\mathfrak{n}^{\prime}$ and $1_{\mathfrak{n}^{\prime}}$ denotes the identity operator on $\mathfrak{n}^{\prime}$.

Proof. As $s$ is an regular element of $G$, it is contained in a Cartan subgroup $\widehat{H}$ of $G$. Let $\widehat{\mathfrak{y}}_{0}$ be the Lie algebra of $\hat{H}$, then its complexification $\hat{\mathfrak{h}}$ is a Cartan subalgebra of g. As is easily seen, $\hat{\mathfrak{h}} \supset \mathfrak{h}^{\prime}$. Therefore $\hat{\mathfrak{h}} \subset \mathfrak{G}$, and hence $\operatorname{Ad} \boldsymbol{h}\left(\mathfrak{n}^{\prime}\right) \subset \mathfrak{n}^{\prime}$ for every $\boldsymbol{h} \in \hat{\mathfrak{h}}$. For every root $\alpha$ of $\{g, \hat{\mathfrak{h}}\}$, let $\boldsymbol{e}_{\alpha}$ be a non-zero element of $g$ such that $\left[\boldsymbol{h}, \boldsymbol{e}_{\alpha}\right]=\alpha(\boldsymbol{h}) \boldsymbol{e}_{\alpha}(\boldsymbol{h} \in \hat{\mathfrak{h}})$. Let $R$ be the set of all roots such that $\boldsymbol{e}_{\alpha} \in \mathfrak{n}^{\prime}$. Then $\mathfrak{n}^{\prime}=\sum_{\alpha \in R} \boldsymbol{C} \boldsymbol{e}_{\alpha}$. Let $\theta$ be the conjugation of $\mathfrak{g}$ associated with the real form $\mathfrak{g}_{0}$. For a root $\alpha$, let $\theta \alpha$ be the root defined by

$$
(\theta \alpha)(\boldsymbol{h})=\overline{\alpha(\theta \boldsymbol{h})} \quad(\boldsymbol{h} \in \hat{\mathfrak{h}}) .
$$

Then $R$ is invariant under $\theta$ because $\mathfrak{n}^{\prime}$ is invariant under $\theta$. And there exists a constant $\tau_{\alpha}$ such that $\theta \boldsymbol{e}_{\alpha}=\tau_{\alpha} \boldsymbol{e}_{\theta \alpha}$ for every root $\alpha$. $\left|\tau_{\alpha}\right|=1$ if $\theta \alpha=\alpha$. Therefore $\sum_{\alpha \in R} y_{\alpha} \boldsymbol{e}_{\alpha} \in \mathfrak{n}_{0}^{\prime}=\mathfrak{n}^{\prime} \cap \mathfrak{g}_{0}$ if and only if $\tau_{\alpha} \bar{y}_{\alpha}=y_{\theta \alpha}(\alpha \in R)$. Since the exponential mapping of $\mathfrak{n}_{0}^{\prime}$ is one-to-one and onto $N^{\prime}$,

$$
\exp \left(\sum_{\alpha \in R} y_{\alpha} \boldsymbol{e}_{\alpha}\right) \in N^{\prime}
$$

if and only if $\tau_{\alpha} \bar{y}_{\alpha}=y_{\theta \alpha}$ for every $\alpha \in R$.

Let $\mathfrak{n}_{1}$ be the center of $\mathfrak{n}^{\prime}$. Then there exists a non-empty subset $R_{1}$ of $R$ such that

$$
\mathfrak{n}_{1}=\sum_{\alpha \in R_{1}} \boldsymbol{C} \boldsymbol{e}_{\alpha}
$$

because if $\alpha, \alpha^{\prime}$, and $\alpha+\alpha^{\prime}$ are roots of $\{\mathfrak{g}, \mathfrak{h}\},\left[\boldsymbol{e}_{\alpha}, \boldsymbol{e}_{\alpha^{\prime}}\right]=N_{\alpha \alpha^{\prime}} \boldsymbol{e}_{\alpha+\alpha^{\prime}}$ where $N_{\alpha \alpha^{\prime}}$ is a non-zero constant. Let $\mathfrak{n}_{2}$ be the inverse image of the center of the quotient $\mathfrak{n}^{\prime} / \mathfrak{n}_{1}$ by the natural mapping of $\mathfrak{n}^{\prime}$ onto $\mathfrak{n}^{\prime} / \mathfrak{n}_{1}$. Then there exists a subset $R_{2}$ of $R$ such that $R_{1} \cap R_{2}=\phi$ and 


$$
\mathfrak{n}_{2}=\sum_{\alpha \in R_{1} \cup R_{2}} \boldsymbol{C} \boldsymbol{e}_{\alpha} .
$$

Inductively, let $\mathfrak{n}_{k}$ be the inverse image of the center of $\mathfrak{n}^{\prime} / \mathfrak{n}_{k-1}$ by the natural mapping of $\mathfrak{n t}^{\prime}$ onto $\mathfrak{n}^{\prime} / \mathfrak{n}_{k-1}$. And let $R_{k}$ be a subset of $R$ such that $R_{k} \cap\left(R_{1} \cup R_{2} \cup \cdots \cup R_{k-1}\right)=\phi$ and

$$
\mathfrak{n}_{k}=\sum_{j=1}^{k} \sum_{\alpha \in R_{j}} \boldsymbol{C} \boldsymbol{e}_{\alpha} \text {. }
$$

Suppose that $\mathfrak{n}^{\prime} / \mathfrak{n}_{r-1}$ is abelian, then $R=R_{1} \cup R_{2} \cup \cdots \cup R_{r}$. Let us line up all roots in $R$ in such a way that any $\alpha \in R_{i}$ is placed in the left of any $\alpha^{\prime} \in R_{j}$ if $i>j$. Fix one of such arrangement $\alpha_{1}, \alpha_{2}$, $\cdots, \alpha_{p}$ and put

$$
n(y)=\exp \left(\sum_{j=1}^{p} y_{i} \boldsymbol{e}_{\alpha_{j}}\right)
$$

where $y=\left(y_{1}, y_{2}, \cdots, y_{p}\right)$. If $n\left(y^{\prime \prime}\right)=n(y) n\left(y^{\prime}\right)$ and $y^{\prime}=\left(y_{1}^{\prime}, y_{2}^{\prime}, \cdots\right.$, $\left.y_{p}^{\prime}\right)$, then for $1 \leqslant j \leqslant p$,

$$
y_{j}^{\prime \prime}=y_{j}+y_{j}^{\prime}+P_{j}\left(y_{1}, y_{2}, \cdots, y_{j-1} ; y_{1}^{\prime}, y_{2}^{\prime}, \cdots, y_{j-1}^{\prime}\right)
$$

where $P_{j}$ is a polynomial of $(2 j-2)$ variables $[8$, p. 82-83].

Since $s \in \widehat{H}$, there exist numbers $a_{j}$ such that $\operatorname{Ad} s\left(\boldsymbol{e}_{\alpha_{j}}\right)=a_{j} \boldsymbol{e}_{\alpha_{j}}$. Then putting $n=n(y)$ and $n^{\prime}=n\left(y^{\prime}\right), n^{\prime}=n^{-1} s n s^{-1}$ is expressed as

$$
n\left(y^{\prime}\right)=n(-y) n\left(a_{1} y_{1}, a_{2} y_{2}, \cdots, a_{p} y_{p}\right) \text {. }
$$

Therefore

$$
\begin{aligned}
& y_{1}^{\prime}=\left(a_{1}-1\right) y_{1} \\
& y_{2}^{\prime}=\left(a_{2}-1\right) y_{2}+P_{2}\left(-y_{1} ; a_{1} y_{1}\right) \\
& y_{p}^{\prime}=\left(a_{p}-1\right) y_{p}+P_{p}\left(-y_{1}, \cdots,-y_{p-1} ; a_{1} y_{1}, \cdots, a_{p-1} y_{p-1}\right) \text {. }
\end{aligned}
$$

Since $\theta$ is identity on $\mathfrak{g}_{0}$ and $G$ is a connected Lie group with Lie algebra $\mathrm{g}_{0}$,

$$
\theta(\operatorname{Ad} g(x))=\operatorname{Ad} g(\theta x)
$$

for any $g \in G, x \in g$. Especially $\theta\left(\operatorname{Ad} s\left(\boldsymbol{e}_{\alpha}\right)\right)=\operatorname{Ad} s\left(\theta \boldsymbol{e}_{\alpha}\right)$ and therefore $a_{i}=\bar{a}_{j}$ if $\alpha_{i}=\theta \alpha_{j}$. Let $R^{\prime}$ be the set of all $\alpha \in R$ such that $\theta \alpha=\alpha$ 
and let $R^{\prime \prime}$ be the set of all $\alpha \in R$ such that $\alpha<\theta \alpha$. If $\alpha \in R^{\prime}$, fix a number $\sigma_{\alpha}$ such that $\sigma_{\alpha}^{-2}=\tau_{\alpha}$. Then $\sigma_{\alpha}^{-2} \bar{y}_{\alpha}=y_{\alpha}$ and hence $\overline{\sigma_{\alpha} y_{\alpha}}=\sigma_{\alpha} y_{\alpha}$, i.e., $\sigma_{\alpha} y_{\alpha}$ is real. Moreover the number $a_{i}$ is real if $\alpha_{i} \in R^{\prime}$.

Introduce new variables $z_{1}, z_{2}, \cdots, z_{p}$ as follows. Put

$$
z_{i}=\sigma_{\alpha_{i}} y_{\alpha_{i}} \text { if } \alpha_{i} \in R^{\prime}, \quad z_{i}=y_{\alpha_{i}} \text { if } \alpha_{i} \in R^{\prime \prime},
$$

and

$$
z_{i}=\left(\tau_{\alpha_{j}}\right)^{-1} y_{\alpha_{i}}=\bar{y}_{\alpha_{j}}=\bar{z}_{j} \quad \text { if } \theta^{-1} \alpha_{i}=\alpha_{j} \in R^{\prime \prime} .
$$

Then the above transformation $y \rightarrow y^{\prime}$ is rewritten as

$$
z_{1}^{\prime}=\left(a_{1}-1\right) z_{1}
$$

and

$$
z_{j}^{\prime}=\left(a_{i}-1\right) z_{i}+P_{i}^{\prime}\left(z_{1}, z_{2}, \cdots, z_{i-1}\right) \quad(2 \leqslant i \leqslant p),
$$

where $P_{i}^{\prime}$ is a polynomial of the variables $z_{1}, z_{2}, \cdots, z_{i-1}$. Put

$$
z_{i}=\xi_{i}+\sqrt{-1} \eta_{i} \quad\left(\xi_{i}, \eta_{i} \in \boldsymbol{R}\right)
$$

for every $\alpha_{i} \in R^{\prime \prime}$. Let us consider the exterior algebra over $\boldsymbol{C}$ generated by $d \xi_{i}, d \eta_{i}$, and $d z_{j}\left(\alpha_{i} \in R^{\prime \prime}, \alpha_{j} \in R^{\prime}\right)$. Then we see easily that

$$
d z_{1}^{\prime} \wedge d z_{2}^{\prime} \wedge \cdots \wedge d z_{p}^{\prime}=\prod_{j=1}^{p}\left(a_{j}-1\right) d z_{1} \wedge d z_{2} \wedge \cdots \wedge d z_{p}
$$

hence

$$
\bigwedge_{\alpha_{i} \in R^{\prime \prime}}\left(d z_{i}^{\prime} \wedge d \bar{z}_{i}^{\prime}\right) \bigwedge_{\alpha \in R^{\prime}} d z_{j}^{\prime}= \pm \prod_{j=1}^{p}\left(a_{j}-1\right) \bigwedge_{\alpha_{i} \in R^{\prime \prime}}\left(d z_{i} \wedge d \bar{z}_{i}\right) \bigwedge_{\alpha \in R^{\prime}} d z_{j} .
$$

This proves that the Jacobian of the transformation

$$
\left(\xi_{i}, \eta_{i}, z_{j}\right) \rightarrow\left(\xi_{i}^{\prime}, \eta_{j}^{\prime}, z_{j}^{\prime}\right) \quad\left(\alpha_{i} \in R^{\prime \prime}, \alpha_{i} \in R^{\prime}\right)
$$

of these $p$ variables is equal to $\pm \prod_{j=1}^{p}\left(a_{j}-1\right)\left(\prod_{j=1}^{p}\left(a_{j}-1\right)\right.$ is real).

Since $s$ is regular, $\prod_{j=1}^{p}\left(a_{j}-1\right) \neq 0$ and therefore the mapping $n \rightarrow n^{\prime}$ is one-to-one regular mapping of $N^{\prime}$ onto itself. And moreover

$$
d n^{\prime}=\left|\prod_{j=1}^{p}\left(a_{j}-1\right)\right| d n .
$$

On the other hand, it is clear that 


$$
\prod_{j=1}^{p}\left(a_{j}-1\right)=\operatorname{det}\left(\operatorname{Ad}_{\mathfrak{n}^{\prime}} s-1_{\mathfrak{n}^{\prime}}\right) .
$$

Moreover

$$
\beta(s)=\left|\prod_{j=1}^{p} a_{j}\right|, \quad D(s)\left(D_{\mathfrak{\$}}(s)\right)^{-1}=\prod_{j=1}^{p}\left(a_{j}-1\right)\left(a_{j}^{-1}-1\right) .
$$

Therefore the lemma is now completely proved.

Applying this lemma to (5.3), we obtain

(5.5) $\quad \operatorname{Sp}\left(T_{x}^{L}\right)$

$$
\begin{gathered}
=\sum_{j=1}^{k} \frac{1}{w_{j}} \int_{\widetilde{K}_{1}} d \hat{u} \int_{N^{\prime}} d n \int_{\widehat{S}^{\prime}} d^{j} \hat{s} \int_{A^{j}} x\left(u^{-1} n^{-1} s^{-1} h s n u\right)\left|D(h) D_{3}(h)\right|^{1 / 2} \tau(h) d^{j} h \\
\left(\widehat{s}=A_{0}^{j} s, \tilde{u}=\Xi_{1} u\right) .
\end{gathered}
$$

The measures $d s, d n$, and $d \tilde{\iota}$ have been taken in such a fashion that for any $\varphi \in C_{0}(G)$,

$$
\begin{aligned}
\int_{G} \varphi(g) d g & =\int_{\widetilde{K}_{1}} d \tilde{u} \int_{N^{\prime}} d n \int_{S_{1}} \varphi(s n u) d s \\
& =\int_{\widetilde{K}_{1}} d \tilde{u} \int_{N^{\prime}} d n \int_{S_{1}} \varphi(n s u) \beta^{-1}(s) d s .
\end{aligned}
$$

Let $d^{j} \tilde{g}$ be an invariant measure on $\Omega^{j}=A_{0}^{j} \backslash G$ such that for $\varphi \in C_{0}(G)$,

$$
\int_{G} \varphi(g) d g=\int_{\Omega^{j}} d^{j} \tilde{g} \int_{A_{j}^{j}} \varphi(h g) d^{j} h \quad\left(\tilde{g}=A_{0}^{j} g \in \Omega^{j}\right) .
$$

Then it follows from (5.2) that for any $\psi \in C_{0}\left(\Omega^{j}\right)$,

$$
\int_{\mathscr{S}^{j}} \psi(\tilde{g}) d^{j} \tilde{g}=\int_{\widetilde{K}_{1}} d \tilde{u} \int_{N^{\prime}} d n \int_{\widehat{S}_{j}} \psi(\operatorname{snu}) d^{j} \hat{\mathcal{S}} \quad\left(\hat{s}=A_{0}^{j} s, \tilde{u}=\Xi_{1} u\right) .
$$

Therefore (5.5) becomes

$$
\operatorname{Sp}\left(T_{x}^{L}\right)=\sum_{j=1}^{k} \frac{1}{w_{j}} \int_{\Omega^{j}} d^{j} \tilde{g} \int_{A^{j}} x\left(g^{-1} h g\right) \sigma(h) d^{j} h
$$

where $\sigma(h)=\left|D(h) D_{\mathfrak{3}}(h)\right|^{1 / 2} \tau(h)$ and $\tilde{g}=A_{0}^{j} g$ for every $j$.

Let $d_{\nu_{j}}$ be an invariant measure on $\mathcal{E}^{j}=Z^{j} \backslash G$ such that

$$
\begin{aligned}
& \int_{G} \varphi(g) d g=\int_{\varepsilon^{j}} d \nu_{j}(\dot{g}) \int_{Z^{j}} \varphi(h g) d^{j} h \\
& =q_{j}^{-1} \int_{\varepsilon^{j}} d_{\nu_{j}}(\dot{g}) \int_{A_{j}^{j}} \varphi(h g) d^{j} h \\
& =p_{j}^{-1} \int_{\varepsilon^{j}} d_{\nu_{j}}(\dot{g}) \int_{H_{j}^{j}} \varphi(h g) d^{j} h \quad\left(\dot{g}=Z^{j} g, \varphi \in C_{0}(G)\right) .
\end{aligned}
$$


Then for any $\psi \in C_{0}\left(\Omega^{j}\right)$, considering it as a function on $\mathcal{E}^{j}$ at the same time,

$$
\int_{2^{j}} \psi(\tilde{g}) d^{j} \tilde{g}=q_{j}^{-1} \int_{\varepsilon^{j}} \psi(\dot{g}) d_{\nu_{j}}(\dot{g}) .
$$

Let $d^{j} \bar{g}$ be an invariant measure on $X^{j}=H_{0}^{j} \backslash G$ which fulfills

$$
\int_{G} \varphi(\bar{g}) d g=\int_{X^{j}} d^{j} \bar{g} \int_{H_{j}^{j}} \varphi(h g) d^{j} h \quad\left(\bar{g}=H_{0}^{j} g, \varphi \in C_{0}(G)\right) .
$$

Then for any $\psi \in C_{0}\left(X^{j}\right)$,

$$
\int_{X^{j}} \psi(\bar{g}) d^{j} \bar{g}=p_{j}^{-1} \int_{\varepsilon^{j}} \psi(\dot{g}) d \nu_{j}(\dot{g}) .
$$

It follows from (5.7) and (5.8) that

$$
\operatorname{Sp}\left(T_{x}^{L}\right)=\sum_{j=0}^{k} \frac{p_{j}}{w_{j} q_{j}} \int_{X^{j}} d^{j} \bar{g} \int_{A^{j}} x\left(g^{-1} h g\right)_{\sigma}(h) d^{j} h,
$$

where in the $j$-th term, $\bar{g}=H_{0}^{j} g$.

Apply Lemma 3.3 to the above integral, then we obtain that

$$
\operatorname{Sp}\left(T_{x}^{L}\right)=\sum_{j=1}^{k} \frac{p_{j}}{w_{j} q_{j}} \int_{G} x(g)|D(g)|^{-1}\left\{\sum_{\omega \subseteq W_{H}^{j}, h_{s}^{\infty j} \in A j} \sigma\left(h_{g}^{\omega}\right)\right\} d g,
$$

where as in Lemma $3.3, h_{\boldsymbol{g}}$ is an element of some $H^{j}$ such that $g=g_{0}^{-1} h_{g} g_{0}$ for some $g_{0} \in G$ and the last summation runs over all $\omega \in W_{H^{j}}$ such that $h_{g}^{\omega} \in A^{j}$, for every $j$.

Thus we arrived at the following theorem.

Theorem 2. Suppose that $S_{1}$ and $L$ fulfill the conditions stated at the beginning of this section. Let $\tau$ and $\pi$ be the characters of $L$ and $T^{L}$ respectively. Then for any $g \in G^{R}$,

$$
\begin{aligned}
\pi(g) & =|D(g)|^{-1 / 2}\left\{\sum_{j=1}^{k} \frac{p_{j}}{w_{j} q_{j}} \sum_{\omega \in W_{H}^{j, \ell_{\circ}^{\omega} \in A j}}\left|D_{\mathfrak{z}}\left(h_{g}^{\omega}\right)\right|^{1 / 2} \tau\left(h_{g}^{\omega}\right)\right\} \\
& =\sum_{j=1}^{k} \frac{p_{j}}{w_{j} q_{j}} \sum_{\omega \in W_{H^{j}, h_{o}^{\omega} \in A j}} \frac{\tau\left(h_{g}^{\omega}\right)}{\prod_{\alpha \in R_{j}}\left|\eta_{\alpha}\left(h_{g}^{\omega}\right)\right|^{-1 / 2}\left|\eta_{\alpha}\left(h_{g}^{\omega}\right)-1\right|}
\end{aligned}
$$

where $R_{j}$ is the set of all positive roots (with respect to some order) of $\left\{\mathfrak{g}, \mathfrak{h}^{j}\right\}$ which are not identically zero on $\mathfrak{h}^{\prime}$. 
Proof. The 1st fart of (5.9) has been already proved. Let us prove the 2rd part. Let $Q_{j}$ be the set of all positive roots of $\left\{\mathfrak{g}, \mathfrak{h}^{j}\right\}$ which are identically zero on $\mathfrak{h}^{\prime}$. If $h \in H^{j}$,

$$
\begin{aligned}
& D(h)=\prod_{\alpha \in Q_{j} \cup R_{j}}\left(\eta_{\alpha}(h)-1\right)\left(\eta_{\alpha}(h)^{-1}-1\right), \\
& D_{\mathfrak{B}}(h)=\prod_{\alpha \in Q_{j}}\left(\eta_{\alpha}(h)-1\right)\left(\eta_{\alpha}(h)^{-1}-1\right) .
\end{aligned}
$$

Hence

$$
|D(h)|^{-1 / 2}\left|D_{\mathfrak{3}}(h)\right|^{1 / 2}=\left\{\prod_{\alpha \in R_{j}}\left|\eta_{\alpha}(h)\right|^{-1 / 2}\left|\eta_{\alpha}(h)-1\right|\right\}^{-1} \text {. Q.E.D. }
$$

Note. Let $\widetilde{H}^{j}\left(\widetilde{A}^{j}\right)$ be the normalizer of $\mathfrak{h}^{j}$ in $G$ (in $\left.S_{1}\right)$. Then $\widetilde{A^{j}} \subset \widetilde{H^{j}}, W_{A^{j}}=\widetilde{A^{j}} / A_{0}^{j}$, and $W_{H^{j}}=\widetilde{H^{j}} / H_{0}^{j}$. The order of $W_{A^{j}}$ is $w_{j}$ by defirition. The order of the subgroup $W^{\prime}=\widetilde{A^{j}} H_{0}^{j} / H_{0}^{j} \cong \widetilde{A}^{j} / Z^{j}$ of $W_{H^{j}}$ is $w_{j} q_{j}$. And the order of $\widetilde{H}^{j} / \widetilde{A^{j}}$ is $p_{j}$ times of that of $W_{H^{j}} / W^{\prime}$.

Remark 1. Let $B$ be the support in $S_{1}$ of the distribution $\tau$. Then that of $\pi$ is contained in the closure of $\cup_{g \in G} g^{-1} B g$.

Remark 2. Any two of the Cartan subgroups $A^{1}, A^{2}, \cdots, A^{k}$ of $S_{1}$ are not conjugate to each other by any inner automorphism of $S_{1}$. But in the set of the Cartan subgroups $H^{1}, H^{2}, \cdots, H^{k}$ of $G$ there may exist the ores which are conjugate to each other by inner automorphisms of $G$. Suppose that $H^{1}, H^{2}, \cdots, H^{d}$ are conjugate to each other, and for $j>d, H^{j}$ is not conjugate to $H^{1}$. Then $G_{H^{1}}=G_{H^{2}}=\cdots$ $=G_{H^{d}}$ and $G_{H^{i}} \cap G_{H^{1}}=\phi$ for $j>d$. Therefore if $g \in G_{H^{1}}$, the first summation in (5.9) rurs over at most on $j=1,2, \cdots, d$ essentially.

Remark 3. If $S$ is the product of its center and its connected component of $e$, then $A_{0}^{j} \subset H_{0}^{j}$ for any $j$. Therefore $q_{j}=1$. In particular, if $G$ is a complex semisimple Lie group, then $p_{j}=q_{j}=1$, and $h \neq h^{\omega}$ for any $h \in H_{j} \cup G^{R}$ and $\omega \in W_{H^{j}}$ different from the identity element (in this case, $k=1$, hence $j=1$ ).

Remark 4. For the universal covering group of $S L(n, \boldsymbol{R})$ $(n \geqslant 3)$, we can find some examples for which $p_{j}>1$ and $q_{j}>1$ for some $j$. 
Note. The conditions put on $S_{1}$ and $L$ at the beginning of this section are sufficient for the deduction of the formula (5.9) but are not necessarily indispensable. In fact, the essential conditions on $S_{1}$ and $L$ for the deduction of the formula (5.9) are the followings.

(I) $S^{0}(D \cap Z) \subset S_{1} \subset S$ and Lemma 3.2 is true for $S_{1}$ and any of its Cartan subgroups, where we employ the same definition of Cartan subgroup as in the case where $S_{1}$ is connected.

(II) $L$ fulfills that

(1) $L_{z}=\lambda(z) 1_{E} \quad(\lambda(z) \in \boldsymbol{C}, z \in E \cap Z)$,

(2) $\operatorname{dim} E(\delta) \leqslant N d(\delta)^{2} \quad(\delta \in \boldsymbol{\omega})$, where $N$ is a constant independent of $\delta$,

(3) the character $\tau$ of $L$ is essentially a function on $S_{1}$ which is locally summable with respect to $d s$.

The connectedness of $G$ is not indispensable for the deduction of the formula (5.9). In fact, let $G$ be the group consisting of all real $n \times n$ matrices whose determinant is equal to 1 or -1 . For this group, the formula (5.9) is true if $L$ verifies the condition (II) above. Because $G$ has an analogous decomposition as that of Iwasawa and Lemma 3.3 is true for $G$, and any $S_{1}$ fulfills the condition (I) above.

Appendix. We keep to the notations of $\S \S 1$ and 2 . Let $T$ be a representation of a connected semisimple Lie group $G$ on a Banach space $\mathcal{H} . \quad T$ is called $K$-finite if $\operatorname{dim} \mathscr{H}(\mathscr{D})<\infty$ for all $\mathscr{D} \in \Omega$.

Lemma. Suppose that $T$ is irreducible. Then $T$ is quasisimple if and only if $T$ is $K$-finite.

Proof. The necessity of the condition is clear from [4(b), Theorem 4]. Therefore it rests only to prove the sufficiency of the condition.

(a) Let us first prove that $T$ is permissible. Let $\mathfrak{A}$ be the set of all bounded operators $A$ which commute with all $T_{g}(g \in G)$. It follows from the irreducibility of $T$ that if $A \in \mathfrak{X}$ and $A \neq 0, A$ is invertible and $A^{-1} \in \mathfrak{A}$. Moreover $\mathfrak{A}$ is a division algebra over. $\boldsymbol{C}$ in 
the natural fashion. Take one $\mathscr{D} \in \Omega$ such that $\mathscr{H}(\mathscr{D}) \neq\{0\}$. Every $A \in \mathfrak{U}$ makes $\mathscr{H}(\mathscr{D})$ invariant and $A \rightarrow A \mid \mathcal{H}(\mathscr{D})$ is a field-isomorphism of $\mathfrak{A}$ into the set of all transformations on $\mathscr{H}(\mathscr{D})$. The latter is of finite dimensions over $\boldsymbol{C}$. Therefore $\mathfrak{A}$ is also finite-dimensional over $\boldsymbol{C}$. Hence $\mathfrak{A}$ is isomorphic to $\boldsymbol{C}$. This means that any $A \in \mathfrak{A}$ is a scalar multiple of the unit operator $1_{\mathscr{H}}$. Thus $T_{z}$ is a scalar multiple of $1_{\mathscr{K}}$ for any $z \in Z$.

(b) Replacing $G$ by its universal covering group if necessary, we may assume that $G$ is simply connected. Let $\Gamma(u)(u \in K)$ be as in $\S 1$. And let $\mu$ be a linear functional on $c_{0}$ such that $T_{z}=e^{\mu\left(r_{(z)}\right)} 1 \mathscr{H}$ $(z \in D \cap Z)$. Then $T_{u}^{\prime}=e^{-\mu(\Gamma(u))} T_{u}$ may be considered as a representation of $K^{*}=K / D \cap Z$. Let $\Omega^{*}$ be the set of all equivalent classes of finite-dimensional irreducible representations of $K^{*}$. For any $\mathscr{D}^{*} \in \Omega^{*}$, let $\mathscr{H}\left(\mathscr{D}^{*}\right)$ be the analogous subspace as $\mathscr{H}(\mathscr{D})$. Let $d\left(\mathscr{D}^{*}\right)$

be the dimension of $\mathscr{D}^{*}$ and $\chi_{D^{*}}\left(u^{*}\right)\left(u^{*} \in K^{*}\right)$ the character of $\mathscr{D}^{*}$. Define

$$
P_{\mathscr{D}^{*}}=d\left(\mathscr{D}^{*}\right) \int_{K^{*}} \chi_{\mathscr{D}^{*}}\left(u^{*}\right) T_{u^{*}}^{*} d u^{*} .
$$

This is a continuous projection of $\mathscr{H}$ onto $\mathscr{H}\left(\mathscr{D}^{*}\right)$.

As is well known, $\mathscr{H}^{\infty}$ (see $\left.\S 2\right)$ is invariant under any $T_{g}(g \in G)$ and any $T_{\boldsymbol{x}}(\boldsymbol{x} \in U(\mathrm{~g}))$. And it is dense in $\mathscr{H}[1]$. Therefore $P_{\mathscr{D}^{*}} \mathscr{H}^{\infty}$ is contained in $\mathscr{H}^{\infty}$ and dense in $\mathscr{H}\left(\mathscr{D}^{*}\right)$. Hence $P_{\mathscr{D}^{*}} \mathscr{H}^{\infty}=\mathscr{H}\left(\mathscr{D}^{*}\right)$ and $\mathscr{H}\left(\mathscr{D}^{*}\right) \subset \mathscr{H}^{\infty}$. Let $\mathscr{H}_{0}$ be the algebraic sum of all $\mathscr{H}(\mathscr{D})(\mathscr{D} \in \Omega)$. Then this is also the algebraic sum of all $\mathscr{H}\left(\mathscr{D}^{*}\right)\left(\mathscr{D}^{*} \in \Omega^{*}\right)$. Therefore $\mathscr{H}_{0} \subset \mathscr{H}^{\infty}$.

$\mathcal{H}_{0}$ is invariant under any $T_{\boldsymbol{x}}(\boldsymbol{x} \in U(\mathfrak{g}))$. And the representation $\boldsymbol{x} \rightarrow T_{\boldsymbol{x}}$ of $U(\mathrm{~g})$ on $\mathcal{H}_{0}$ is algebraically irreducible. In fact, it can be easily shown that Theorem 16 in $[9$, p. 545] is also valid for our case, although $K$ is not necessarily compact. Therefore we see as in (a) that

$$
T_{z} v=\alpha(\boldsymbol{z}) v \quad\left(\boldsymbol{z} \in 3, v \in \mathcal{H}_{0}\right)
$$

where $\alpha$ is a homomorphism of $B$ into $\boldsymbol{C}$. 
(c) Let us introduce in $\mathscr{H}^{\infty}$ the topology defined in [1, p. 115]. Then $\mathcal{H}^{\infty}$ becomes Fréchet space. $T_{g}(g \in G)$ defines a representation of $G$ on $\mathscr{H}^{\infty}$ and every element of $\mathscr{H}^{\infty}$ is differentiable under this representation [1, p. 115]. Consider the representation $u^{*} \rightarrow T_{u^{*}}^{\prime}$ $\left(u^{*} \in K^{*}\right)$ on $\mathcal{H}^{\infty}$ and apply Lemma 4 of $[4(\mathrm{f})]$ on it. Then we see that $\mathcal{H}_{0}$ is dense in $\mathcal{H}^{\infty}$. On the other hand, $T_{\boldsymbol{x}}(\boldsymbol{x} \in U(\mathfrak{g}))$ are all continuous in $\mathcal{H}^{\infty}$. Therefore it follows from (1) that

$$
T_{z} v=\alpha(\boldsymbol{z}) v \quad\left(\boldsymbol{z} \in \mathfrak{g}, v \in \mathcal{H}^{\infty}\right) .
$$

This completes the proof of the sufficiency of the condition. Q.E.D.

Note. Let $T$ be not necessarily irreducible. Suppcse that $T$ is permissible and $K$-finite. Then if $T$ has the property (1), it has also the property (2) and vice versa. In fact, (b) and (c) in the above prcof are also valid for this case without any modification.

\section{References}

[1] F. Bruhat, Sur les représentations induites des groupes de Lie, Bull. Soc. Math. France, 84 (1956), 97-205.

[2] M. I. Graev, Unitary representations of real simple Lie groups (in Russian), Trudy Mosk. Mat. Ob., 7 (1958), 335-389.

[3] I. M. Gel'fand and M. I. Graev, Traces of unitary representations of real unimodular groups (in Russian), Doklady Acad. Nauk, 100 (1955), 1037-1040.

[4] Harish-Chandra,

(a) Representations of a semisimple Lie group on a Banach space. I, Trans. Amer. Math. Soc., 75 (1953), 185-243.

(b) Representations of semisimple Lie groups. III, ibid., 76 (1954), 234-253.

(c) The Plancherel formula for complex semisimple Lie groups, ibid., 76 (1954), 485-528.

(d) The characters of semisimple Lie groups, ibid., 83 (1956), 98-163.

(e) Invariant eigendistributions on semisimple Lie groups, ibid., 119 (1965), 457-508.

(f) Discrete series for semisimple Lie groups. II. Acta Math., 116 (1966), 1-111.

[5] T. Hirai,

(a) The characters of irreducible representations of the Lorentz group of $n$-th order, Proc. Japan Acad., 41 (1965), 526-531.

(b) Classification and the characters of irreducible representations of $S U(p, 1)$, ibid., 42 (1966), 907-912.

(c) Invariant eigendistributions on real simple Lie groups. I, to appear in J. Math. Kyoto Univ.

(d) The Plancherel formula for $S U(p, q)$, forthcoming. 
(e) The characters of some induced representations of semisimple Lie groups, to appear in Proc. Japan Acad.

[6] I. E. Segal, Hypermaximality of certain operators on Lie groups, Proc. Amer. Math. Soc., 3 (1952), 13-15.

[7] M. Sugiura, Conjugate classes of Cartan subalgebras in real semisimple Lie algebras, J. Math. Soc. Japan, 11 (1959), 374-434.

[8] L. Pukanszky, Leçon sur les représentations des groupes, Dunod, Paris, 1967.

[9] R. Godement, A theory of spherical functions. I, Trans. Amer. Math. Soc., 73 (1952). 496-556.

[10] I. Ya. Vakhutinskiî, Unitary representations of the group $G L(3, \boldsymbol{R})$ of real non degenerate matrices of order three, Doklady Akad. Nauk SSSR, 170 (1966). 21-22.

[11] L. S. Pontrjagin, Continuous groups, Moscow, 1954 (in Russian).

[12] S. Helgason, Differential geometry and symmetric spaces, Academic Press, 1962.

Kyoto UnIVERSITY 